\title{
Axially equilibrated displacement-based beam element for simulating the cyclic inelastic behaviour of RC members
}

\author{
Danilo Tarquini ${ }^{1, *}{ }^{\dagger}$ (D), João P. Almeida ${ }^{2}$ (D) and Katrin Beyer ${ }^{3}$ \\ ${ }^{1}$ Earthquake Engineering and Structural Dynamics Laboratory (EESD), School of Architecture, Civil and Environmental \\ Engineering (ENAC), École Polytechnique Fédérale de Lausanne (EPFL), EPFL ENAC IIC EESD, GC B2 495, Station \\ 18, CH-1015 Lausanne, Switzerland \\ ${ }^{2}$ Earthquake Engineering and Structural Dynamics Laboratory (EESD), School of Architecture, Civil and Environmental \\ Engineering (ENAC), École Polytechnique Fédérale de Lausanne (EPFL), EPFL ENAC IIC EESD, GC B2 484, Station \\ 18, CH-1015 Lausanne, Switzerland \\ ${ }^{3}$ Earthquake Engineering and Structural Dynamics Laboratory (EESD), School of Architecture, Civil and Environmental \\ Engineering (ENAC), École Polytechnique Fédérale de Lausanne (EPFL), EPFL ENAC IIC EESD, GC B2 504, Station \\ 18, CH-1015 Lausanne, Switzerland
}

\begin{abstract}
SUMMARY
Distributed plasticity beam elements are commonly used to evaluate limit state demands for performancebased analysis of reinforced concrete (RC) structures. Strain limits are often preferred to drift limits because they directly relate to damage and are therefore less dependent on member geometry and boundary conditions. However, predicting accurately strain demands still represents a major simulation challenge. Tension shift effects, which induce a linear curvature profile in the plastic hinge region of RC columns and walls, are one of the main causes for the mismatch between experimental and numerical estimates of local level quantities obtained through force-based formulations. Classical displacement-based approaches are instead suitable to simulate such linear curvature profile. Unfortunately, they verify equilibrium only on an average sense due to the wrong assumption on the axial displacement field, leading to poor deformation and force predictions. This paper presents a displacement-based element in which axial equilibrium is strictly verified along the element length. The assumed transversal displacement field ensures a linear curvature profile, connecting accurately global displacement and local strain demands. The proposed finite element is validated against two sets of quasi-static cyclic tests on RC bridge piers and walls. The results show that curvature and strain profiles for increasing ductility demands are significantly improved when axially equilibrated rather than classical displacement-based or force-based elements are used to model the structural members. Copyright (C) 2017 John Wiley \& Sons, Ltd.
\end{abstract}

Received 20 July 2016; Revised 17 November 2016; Accepted 29 November 2016

KEY WORDS: beam element; axial equilibrium; displacement-based formulation; distributed plasticity; tension shift effects

\section{INTRODUCTION}

Performance-based assessment of structures is based on the definition of clear limit states, from which economical losses can be estimated for various ground motion intensities. Limit states can be based either on global element drift/chord rotation limits or on local material strain limits, which are deemed to be a better indicator of structural damage [1]. The difficulty in defining element-related drift/chord rotation limits for bi-directional loading is not faced if material strain

*Correspondence to: Danilo Tarquini, Earthquake Engineering and Structural Dynamics Laboratory (EESD), School of Architecture, Civil and Environmental Engineering (ENAC), Ecole Polytechnique Fédérale de Lausanne (EPFL), EPFL ENAC IIC EESD, GC B2 495, Station 18, CH-1015 Lausanne, Switzerland.

†E-mail: danilo.tarquini@epfl.ch 
demands can be directly computed and verified. In reinforced concrete (RC) structures, damage concentrates in plastic hinge regions, and therefore efficient models should accurately simulate the main sources of deformation associated to the plastic hinge development. The classical interpretation of such mechanism, at least for well-detailed members, considers three main components, namely the moment gradient, tension shift and anchorage slip (or strain penetration) effects [2].

Although several modelling approaches are available, the attractive compromise between accuracy and computational cost renders beam element models one of the most widely employed numerical tools in engineering practice, especially when complex and multi-member structures such as buildings or bridges are involved. Beam element models are typically divided into lumped and distributed plasticity approaches. The former are more performant from the computational viewpoint as they typically lump the three above mentioned components of the plastic hinge at pre-defined member locations. This is accomplished by using the concept of equivalent plastic hinge length, which therefore often features three terms [2]. On the contrary, each of those terms can be individuated in the so-called distributed plasticity elements. Anchorage slip can be accounted for by a zero-length element, such as that developed by Zhao and Sritharan [3] or any other appropriately calibrated relation $[4,5]$. The moment gradient is explicitly simulated because the development of plasticity is not restrained to a specific member location but can spread along several integration points (IPs) in which the finite element (FE) is typically discretized. Up to the present moment, tension shift effects due to inclined cracking caused by shear force-see Figure 1(a) - have not been explicitly addressed in the pre-peak phase of the force-displacement member response, although their influence will necessarily affect the comparison between experimental and numerical results at the global (i.e. member displacement) level $[6,7]$. In the post-peak branch, the need to use a regularization length makes it possible to indirectly account for them [8,9]. This paper shows that models based on distributed plasticity elements and classical beam theory can also be adapted to directly incorporate tension shift effects whilst verifying strictly axial equilibrium, thus significantly strengthening the accuracy of these approaches at the local scale.

Put simply, distributed plasticity elements can be mainly subdivided in displacement-based (DB) and force-based (FB) formulations [10] depending on the type of the imposed independent fields. As

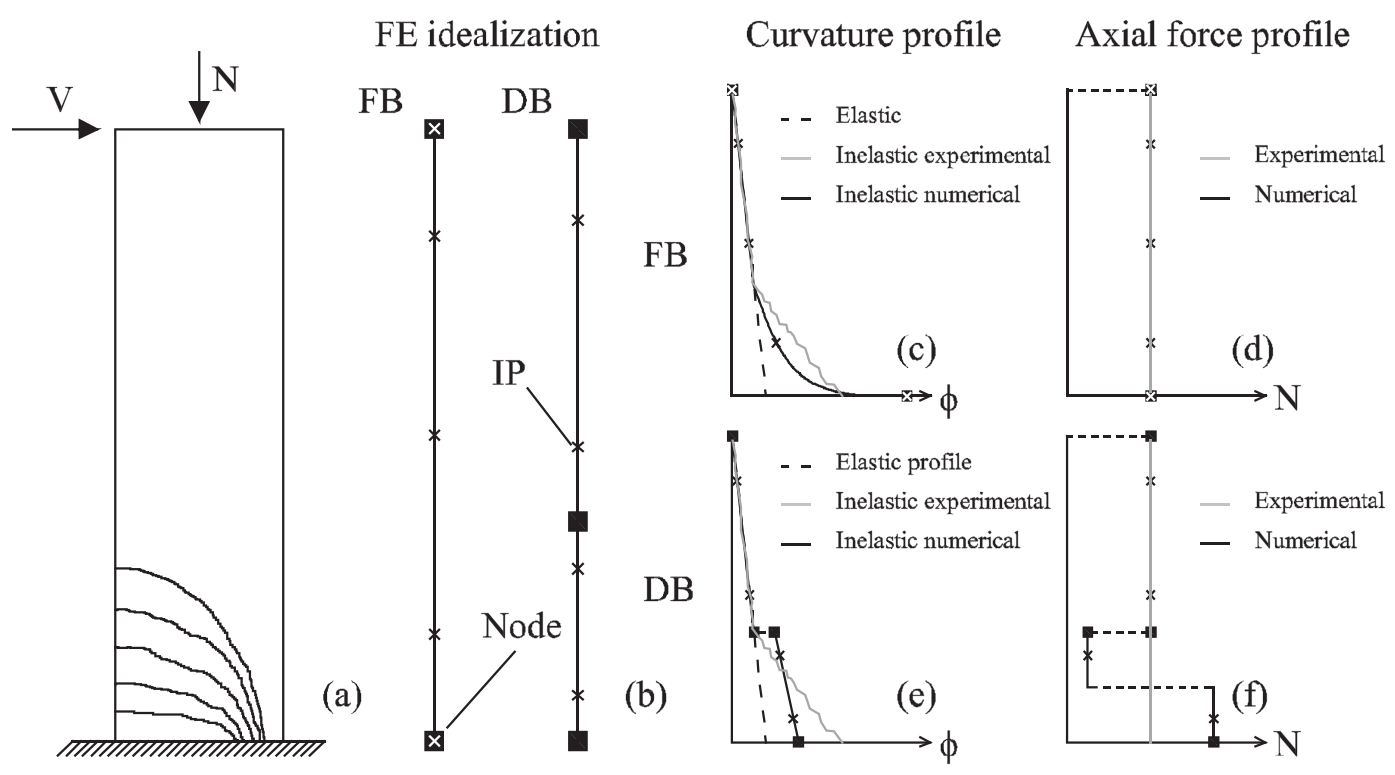

Figure 1. RC member subjected to top vertical and horizontal load: (a) qualitative sketch of inclined cracks due to tension shift effects; (b) structural discretization with FB and DB elements; qualitative experimental versus numerical curvature and axial strain profiles: FB element models-(c) and (e); DB element models(d) and (f). 
their name suggest, displacement and force distributions along the element length are assigned in the former and latter case. The hypotheses governing the beam kinematics control the number of sectional deformations that arise. The current work considers only Euler-Bernoulli (EB) beam hypothesis because: (i) it is simple and allows to model most of the structural members in a RC structure; (ii) linear and nonlinear EB beam elements are available in roughly all the commonly used structural analysis software (e.g. [11, 12]). Linear and Hermitian polynomial functions are employed in classical DB formulations to characterize axial and transversal displacement fields. These assumptions provide an exact solution only for linear elastic material and nodal loads. On the other hand, constant and linear shape functions are used to define axial force and bending moment distributions in FB formulations, which results in an exact solution regardless of the development of material nonlinear response. Additionally, in FB formulations, equilibrium is strictly verified along the element length — see Figure 1(d) — whereas in DB approaches, equilibrium is only verified in an average sense [13] — see Figure 1(f). As a consequence, whilst a single FB element usually suffices to simulate the nonlinear response of a structural member, member discretization in several FEs is required if DB elements are used-see Figure 1(b).

The state determination of DB formulations is simpler and less computationally demanding than their FB counterpart as the element end forces and tangent stiffness matrix are directly obtained by integration of the sectional responses, hence avoiding the intra-element iterations needed for FB approaches. However, the superiority of the latter in terms of theoretical accuracy and significant size reduction of the resulting global structural stiffness matrix has led to a gradual reduction in the use of DB formulations over the past 15-20 years.

More recently, experimental measurements from accurate instrumentation systems [14] applied to nonlinearly responding RC members have confirmed important limitations of the FB formulations. Disregarding the effects of tension shift in the pre-peak phase was proven to be one of the most relevant. The latter cause a linear distribution of plastic curvatures inside the plastic zone of the structural member ([15-18], see Figure 1), and, as pointed out by Priestley et al. [2], it represents the first reason for the mismatch between the force-displacement response as obtained from a FB element (which verifies equilibrium in an exact form) and experimental results. Furthermore, the previously mentioned test campaign [14] has shown that the intersection between plastic and elastic curvature profiles occurs at an increasing height for larger ductility demands.

DB formulations offer a solution for the analytical simulation of the above physical phenomena and thus provide a bypass to the limitations brought about by FB approaches. In fact, the observed linear curvature profiles in the plastic hinge region of RC members can be simulated by imposing appropriate lateral displacement fields to the beam FE, which is the natural framework of DB and not FB formulations-see Figure 1(c) and (e). This paper represents a first step to reflect the discussed experimental findings in beam element models with a view to predict more confidently the performance of RC structures. To accomplish such goal, a fundamental drawback of the classical DB formulations is addressed beforehand. As already mentioned, the imposed linear axial displacement field implies that axial equilibrium is only verified in an average sense, which results in case of material nonlinearity in different values of the axial force for distinct integration sections. This leads to a misevaluation of the moment capacity of the structural member and therefore to a poor local and global performance of the FE [13]. In this paper, an enhanced DB element for the inelastic simulation of RC members is proposed in which the axial equilibrium is strictly verified (hence emulating the advantages of a FB formulation in this respect) through the use of an iterative procedure. It will be shown that the use of such an element, combined with a convenient structural discretization, leads to an important improvement in the simulation of global and, more importantly, local level quantities when compared with models employing classical DB or FB approaches.

The new element and its state determination are described in Section 2 along with an application example describing its main features and relative performance with respect to classical DB formulations. Section 3 benchmarks the performance of the new beam element against two sets of experimental tests on RC bridge piers and RC walls. Comparison at the global and local levels, namely curvature and strain profiles, are provided and limitations of the proposed formulations discussed. Conclusions are drawn in Section 4. 


\section{AXIALLY EQUILIBRATED DISPLACEMENT-BASED ELEMENT: FORMULATION AND STATE DETERMINATION}

The beam formulation herein proposed is a plane frame element, which implies that in the global reference system $(X, Y)$ six components are required to characterize the vector of nodal forces $(\mathbf{P})$ and displacements $(\mathbf{U})$. Three components suffice instead to describe the basic forces and displacements $\left(\mathbf{p}^{b s c}=\left[p_{1}^{b s c}, p_{2}^{b s c}, p_{3}^{b s c}\right]^{T}\right.$ and $\left.\mathbf{u}^{b s c}=\left[u_{1}^{b s c}, u_{2}^{b s c}, u_{3}^{b s c}\right]^{T}\right)$ in the member-bound reference system without rigid-body modes $(x, y)$, which are shown in Figure 2.

The concept of a DB element satisfying axial equilibrium was originally proposed by Izzudin et al. [19] for nonlinear-elastic problems. No plasticity was considered in their work, and explicit expressions were derived for both sectional forces and stiffness matrix. Further, shape functions were not defined for the axial displacement field while a quartic formulation was used for the transversal displacement field. The element end forces $\mathbf{p}^{b s c}$ and tangent stiffness matrix $\mathbf{K}$ were then obtained according to the principle of virtual work and by direct differentiation of each individual component, respectively.

The formulation presented in this manuscript features the following distinctive aspects with respect to the one above: (i) material constitutive laws including plastic deformations and cyclic behaviour were considered in the derivation and validation phases; (ii) Hermitian polynomials for the transversal displacement field are used, which results in linear curvature profiles as in classical DB approaches. This assumption, as already discussed, is sought in order to numerically account for tension shift effects; and (iii) a variational approach is employed to determine the element end forces and the tangent stiffness matrix. In particular, differences were obtained with respect to [19] in the components of the tangent stiffness matrix referring to the derivatives of the basic axial force $p_{1}^{b s c}$ with respect to the vector of basic displacements $\mathbf{u}^{b s c}$.

A similar procedure to the one proposed by Izzudin et al. [19] was used to achieve axial equilibrium of the element. The latter is discussed in subsection 2.1, while subsection 2.2 focuses on the state determination algorithm. Finally, in subsection 2.3, the main features of the proposed FE formulation are explored, and the new beam element is compared against classical DB formulations with the aid of an application example.

\subsection{Axial equilibrium}

In a beam subjected to nodal loads, equilibrium considerations impose the axial force $N(x)$ to be constant along the beam axis $x$ and equal to the nodal axial basic force $p_{1}^{b s c}$. As discussed in Section 1, this is not the case for classical DB elements employing nonlinear material constitutive laws. In fact, the linear shape function approximating the axial displacement field $u(x)$ ensures axial equilibrium only in an average sense, which yields different values of the axial force at distinct IPs (i.e. $N^{I P j} \neq N^{I P j+1}$ ).

The main idea behind the axial equilibrium procedure consists in correcting the set of sectional axial strains $\varepsilon_{0}^{I P j}$ such that the value of the axial force is the same in all IPs $\left(N^{I P j}=N^{I P j+1}\right)$. Given the intrinsic nonlinearity of the problem, an iterative procedure is required to attain this goal, which is discussed in the following paragraphs and schematically represented in the flowchart of Figure 3.

Consider a Newton-Raphson (NR) cycle $n$ within an arbitrary load step $l$, for which a vector of displacement increments in the basic reference system $\Delta \mathbf{u}^{b s c}$ is imposed. The steps to be performed

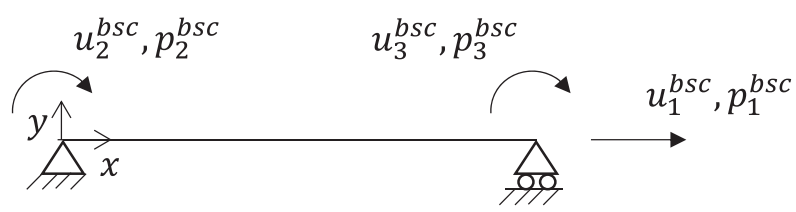

Figure 2. Element forces and displacements in the basic reference system. 


\section{AXIALLY EQUILIBRATED DISPLACEMENT-BASED ELEMENT FOR RC MEMBERS}

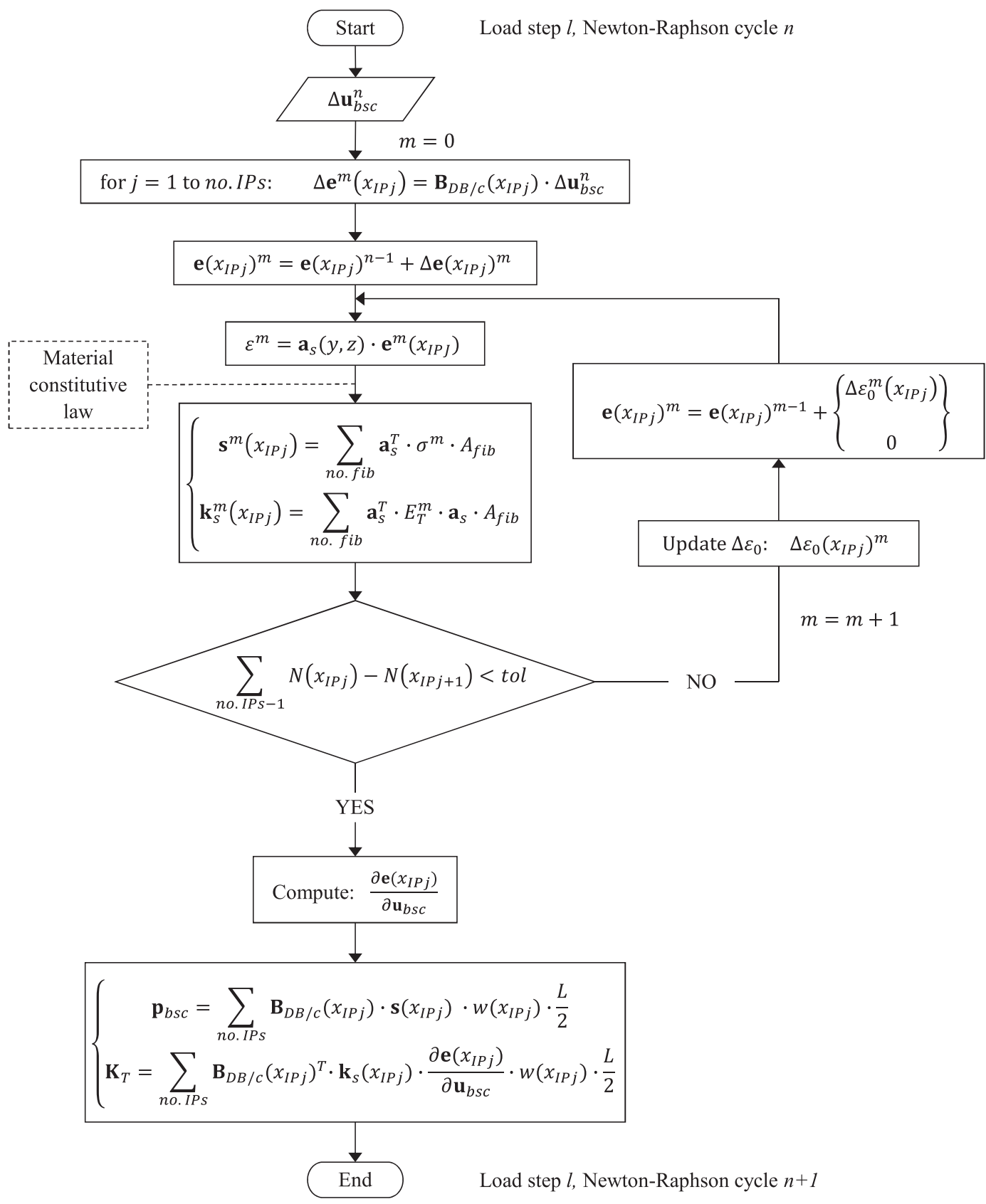

Figure 3. Flowchart for the element state determination of the axially equilibrated displacement-based element.

to go from the basic to the global reference system require classical structural analysis operations, i.e. the rotation of the coordinate system as well as the use of linear or nonlinear compatibility and equilibrium relations [20], and will thus be omitted in this work. Shape functions employed in classical DB formulations (linear and Hermitian polynomials for the axial and transversal displacement fields) are initially used to obtain, after differentiation, the corresponding increments of sectional deformations $\triangle \mathbf{e}^{I P J}$ at all IPs along the element. The sectional deformations are calculated from the basic nodal displacements through pre-multiplication by the matrix $\mathbf{B}_{D B / c}$, where the subscript $D B / c$ underlines that classical DB shape functions are considered. Once the sectional axial strain and curvature increments are known $\left(\Delta \varepsilon_{0}^{I P j}\right.$ and $\left.\Delta \phi^{I P j}\right)$, the EB hypothesis and the sectional constitutive law enable to compute the generalized sectional forces $\mathbf{s}^{I P j}$. The differences between the 
axial forces recorded in two successive IPs are then calculated. Axial equilibrium is considered to be satisfied if the cumulative sum of these differences throughout all pairs of IPs in the element is below a certain tolerance. It is noted that this convergence criterion is different from the one proposed by Izzudin et al. [19], where strain differences were checked. If such axial equilibrium is verified, the state determination proceeds as for the classical DB formulation; otherwise, intraelement iterations (identified with the index $m$ in Figure 3) on the increment of sectional axial strains at each IP $\left(\Delta \varepsilon_{0}^{I P j}\right)$ are performed.

Two conditions need to be fulfilled: (i) the axial force should be equal in all IPs, and (ii) the integral of the axial strains along the element length must correspond to the basic axial displacement $u_{1}^{b s c}$. They are expressed in equations (1) and (2), respectively:

$$
\left\{\begin{array}{l}
N^{I P j}+k_{11}^{I P j} \cdot \Delta \varepsilon_{0}^{I P j}=N^{I P j+1}+k_{11}^{I P j+1} \cdot \Delta \varepsilon_{0}^{I P j+1} \quad \text { for } j=1 \text { to no. IPs }-1 \\
\sum_{j=1}^{n o \cdot I P S} \frac{w^{I P j} \cdot L}{2} \cdot\left(\varepsilon_{0}^{I P j}+\Delta \varepsilon_{0}^{I P j}\right)=u_{1}^{b s c}
\end{array}\right.
$$

where $k_{11}^{I P j}$ is the first row-first column component of the sectional stiffness matrix evaluated at $I P_{j}, w^{I P j}$ is the integration weight of the $j^{\text {th }}$ IP (it is assumed that the sum of the integration weights is equal to 2) and $L$ is the element length. As it can be noticed, conditions (1) and (2) provide a linear system of equations; the number of equations corresponds to the number of IPs. The equations can be solved at each IP to obtain the axial strain increments $\Delta \varepsilon_{0}^{I P j}$ as function of the current set of axial forces. These relationships are provided in equations (3) and (4) for the first and the remaining IPs:

$$
\begin{gathered}
\Delta \varepsilon_{0}^{I P_{1}}=\frac{\frac{2 \cdot u_{1}^{b s c}}{L}-\sum_{j=1}^{n o . I P s} w^{I P j} \cdot\left(\varepsilon_{0}^{I P_{j}}+\frac{N^{I P_{1}}-N^{I P_{j}}}{k_{11}^{I P_{j}}}\right)}{\sum_{j=1}^{n o . I P s} w^{I P j} \cdot \frac{k_{11}^{I P_{1}}}{k_{11}^{I P_{j}}}} \\
\Delta \varepsilon_{0}^{I P_{j}}=\frac{\left(N^{I P_{1}}-N^{I P_{j}}\right)+k_{11}^{I P_{1}} \cdot \Delta \varepsilon_{0}^{I P_{1}}}{k_{11}^{I P_{j}}}
\end{gathered}
$$

The so computed strain increments $\Delta \varepsilon_{0}^{I P_{j}}$ are used to update the total axial strains $\varepsilon_{0}^{I P_{j}}$ from the previous intra-element iteration, and new sectional forces are computed. As shown in Figure 3, the procedure is repeated until the resulting axial forces $N^{I P_{j}}$ are equilibrated. In other words, this internal iterative procedure corrects the constant axial strain profile as obtained from the classical DB approach to ensure the same value of the axial force along the element. Note that the curvature profile remains linear as imposed in classical DB formulations.

\subsection{Element state determination}

The present subsection discusses the state determination for the axially equilibrated DB element, which consists in the evaluation of the element end forces $\mathbf{p}^{b s c}$ and tangent stiffness matrix $\mathbf{K}^{b s c}$ for a given increment of basic displacements $\Delta \mathbf{u}^{b s c}$. Once the axial force is equilibrated according to the method previously discussed, the generalized deformations $\mathbf{e}^{I P_{j}}$ at a generic IP can be decomposed as:

$$
\mathbf{e}^{I P j}=\mathbf{B}_{D B / c}^{I P j} \cdot \mathbf{u}^{b s c}+\mathbf{e}_{A E}^{I P j} \quad \text { with } \quad \mathbf{e}_{A E}^{I P j}=\left\{\begin{array}{c}
\varepsilon_{0, A E}^{I P j} \\
\\
0
\end{array}\right\}
$$

where the first term $\mathbf{B}_{D B / c}^{I P j} \cdot \mathbf{u}^{b s c}$ represents the contribution associated to the classical DB shape functions, while the second $\mathbf{e}_{A E}^{I P j}$ corresponds to the sum of the incremental corrections of the sectional axial strain computed during the internal iterative process, identified as $\varepsilon_{0, A E}^{I P j}$ in equation (5). 


\section{AXIALLY EQUILIBRATED DISPLACEMENT-BASED ELEMENT FOR RC MEMBERS}

The element end forces are determined by application of the principle of virtual displacements (PVD), which can be written as follows:

$$
\left[\delta \mathbf{u}^{b s c}\right]^{T} \cdot \mathbf{p}^{b s c}=\int_{L}[\delta \mathbf{e}(x)]^{T} \cdot \mathbf{s}(x) d x
$$

The numerical integration of the expression above over the total number of IPs yields:

$$
\left[\delta \mathbf{u}^{b s c}\right]^{T} \cdot \mathbf{p}^{b s c}=\sum_{n o . I P S}\left[\delta \mathbf{e}^{I P_{j}}\right]^{T} \cdot \mathbf{s}^{I P_{j}} \cdot w^{I P_{j}} \cdot \frac{L}{2}
$$

For the axially equilibrated DB element, the PVD can be specialized by using equations (5) and (7):

$$
\left[\delta \mathbf{u}^{b s c}\right]^{T} \cdot \mathbf{p}^{b s c}=\left[\delta \mathbf{u}^{b s c}\right]^{T} \cdot \sum_{n o . I P s}\left[\mathbf{B}_{D B / c}^{I P_{j}}\right]^{T} \cdot \mathbf{s}^{I P_{j}} \cdot w^{I P_{j}} \cdot \frac{L}{2}+\sum_{n o . I P s}\left[\delta \mathbf{e}_{A E}^{I P_{j}}\right]^{T} \cdot \mathbf{s}^{I P_{j}} \cdot w^{I P_{j}} \cdot \frac{L}{2}
$$

The previous equations should be valid for any virtual increment $\delta$, which results in the following system of two equations that have to be satisfied simultaneously:

$$
\left\{\begin{array}{c}
\mathbf{p}^{b s c}=\sum_{n o .} \sum_{I P S}\left[\mathbf{B}_{D B / c}^{I P_{j}}\right]^{T} \cdot \mathbf{s}^{I P_{j}} \cdot w^{I P_{j}} \cdot \frac{L}{2} \\
\sum_{n o .}\left[\delta \mathbf{e}_{A E}^{I P_{j}}\right]^{T} \cdot \mathbf{s}^{I P_{j}} \cdot w^{I P_{j}} \cdot \frac{L}{2}=0
\end{array}\right.
$$

The verification of equation (2) directly demonstrates equation (10) for any $\delta \mathbf{e}_{A E}^{I P_{j}}$, while equation (9) shows that the basic end forces $\mathbf{p}^{b s c}$ are computed from the internal section forces $\mathbf{s}(x)$ as in classical DB formulations.

The element tangent stiffness matrix $\mathbf{K}^{b s c}$ is then straightforwardly obtained by deriving the element end forces $\mathbf{p}^{b s c}$ with respect to the element basic displacements $\mathbf{u}^{b s c}$. With the aid of the chain rule of derivation and considering that the matrix $\mathbf{B}_{D B / c}$ does not depend on $\mathbf{u}^{b s c}$, the following equation is derived:

$$
\mathbf{K}^{b s c}=\frac{\partial \mathbf{p}^{b s c}}{\partial \mathbf{u}^{b s c}}=\sum_{n o .}{ }_{I P s}\left[\mathbf{B}_{D B / c}^{I P_{j}}\right]^{T} \cdot \frac{\partial \mathbf{s}^{I P_{j}}}{\partial \mathbf{e}^{I P_{j}}} \cdot \frac{\partial \mathbf{e}^{I P_{j}}}{\partial \mathbf{u}^{b s c}} \cdot w^{I P_{j}} \cdot \frac{L}{2}
$$

where the partial derivatives of the sectional forces with respect to generalized strains $\partial \mathbf{s}^{I P_{j}} / \partial \mathbf{e}^{I P_{j}}$ correspond, by definition, to the sectional stiffness matrix $\mathbf{k}$. The partial derivatives $\partial \varepsilon_{0}^{I P_{j}} / \partial \mathbf{u}^{b s c}$ at each IP can be calculated from equations (3) and (4), making additional use of the conditions expressed in (1) and (2):

$$
\begin{aligned}
& \frac{\partial \varepsilon_{0}^{I P_{1}}}{\partial u_{k}^{b s c}}=\frac{\frac{\partial}{\partial u_{k}^{s s c}}\left(\frac{2 \cdot u_{1}^{b s c}}{L}\right)-\sum_{j=1}^{n o . I P S} w^{I P j} \cdot\left(\frac{k_{12}^{I P_{1}} \frac{\partial \phi^{I P_{1}}}{\partial u_{k}^{b s c}}-k_{12}^{I P_{j}} \frac{\partial \phi^{I P j}}{\partial u_{k}^{b_{c}}}}{k_{11}^{I P_{j}}}\right)}{\sum_{j=1}^{n o . I P_{s}} w^{I P j} \cdot \frac{k_{11}^{I P_{1}}}{k_{11}^{I_{j}}}} \\
& \frac{\partial \varepsilon_{0}^{I P_{j}}}{\partial u_{k}^{b s c}}=\frac{\left(k_{12}^{I P_{1}} \cdot \frac{\partial \phi^{I P_{1}}}{\partial u_{k}^{b s c}}-k_{12}^{I P_{j}} \cdot \frac{\partial \phi^{I P P_{j}}}{\partial u_{k}^{b s c}}\right)+k_{11}^{I P_{1}} \cdot \frac{\partial \varepsilon_{0}^{I P_{1}}}{\partial u_{k}^{b s c}}}{k_{11}^{I P_{j}}}
\end{aligned}
$$

where the subscript $k$ is used to indicate the component of $\mathbf{u}^{b s c}$ with respect to which the derivation is performed. The derivatives of the curvatures are not presented here because they are similar to those obtained from classical DB approaches. The state determination procedure summarized in the flowchart of Figure 3 depicts the application of the expressions presented in the current subsection. 


\subsection{Axially equilibrated versus classical DB element}

The axially equilibrated DB (DB/ae) formulation was implemented in the finite element software SAGRES (Software for Analysis of GRadient Effects in Structures), which also includes in its library classical $\mathrm{DB}(\mathrm{DB} / \mathrm{c})$ and $\mathrm{FB}$ elements [21]. In this subsection, the main features of the proposed FE are presented resorting to an application example. Namely, models using DB/ae and $\mathrm{DB} / \mathrm{c}$ elements are compared both at the global and local level in order to highlight their relative advantages and drawbacks.

A 3-m RC cantilever column, subjected to an axial load ratio of about $1.25 \%$ and an incremental lateral displacement $\Delta$, served as reference structure. The RC section was $300 \mathrm{~mm}$ large and $400 \mathrm{~mm}$ deep (bending direction), and 20-mm concrete cover was assumed in both directions. The longitudinal reinforcement was formed by $16 \varnothing 10-\mathrm{mm}$ steel bar corresponding roughly to a reinforcement ratio of about $1 \%$. A schematic representation of the reference structure as well as of the sectional discretization used in all FE models herein considered is shown in Figure 4(a). The mathematical relation proposed by Popovics [22] was used for both confined and unconfined concrete $\left(E_{c}=30 \mathrm{GPa}, f^{\prime}{ }_{c}=37 \mathrm{MPa}, \varepsilon_{c}=0.002, f^{\prime}{ }_{c c}=42 \mathrm{MPa}, \varepsilon_{c c}=0.003\right)$, while a bilinear constitutive law was assigned to the longitudinal reinforcement $\left(E_{s}=200 \mathrm{GPa}, f_{y}=480 \mathrm{MPa}, b=0.005\right)$.

The influence of mesh refinement on the global-level response is investigated in Figure 4(b), which displays pushover curves, in the form of a dimensionless lateral resisting force $V_{N}=\left(V \times L_{s}\right) /(N \times h)$ versus horizontal drift $\Delta / L_{s}$. Models employing one, two and three DB elements (both DB/ae and $\mathrm{DB} / \mathrm{c}$ ), as well as a single FB element, were considered. DB elements featured two Gauss-Legendre IPs, while five Gauss-Lobatto IPs were used for the FB element model. As discussed in Calabrese et al. [13], FB elements are sensitive to the element discretization, and a minimum number of four IPs is generally required for good-accuracy solutions under strain hardening response. DB/c element models, on the other hand, are only sensitive to the structural discretization, and hence it is not justifiable to use more than two IPs per element. For comparison purposes, the same number of IPs is used as well for DB/ae elements, even though, as discussed below with respect to Figure 4(c), a larger number would be required for a closer-to-objective response.

The model with a single DB/c element shows, as expected, the strongest and stiffest response due to the constraints imposed in both the axial and transversal displacement fields. By removing the constraint on the axial displacement field through the iterative procedure introduced in subsection 2.1, the model using one DB/ae element provides a considerably softer response, causing a reduction in the simulated lateral strength. However, it can be noted that the latter is still overestimated when compared to the solution provided by the FB formulation, where no displacement fields are assigned and exact equilibrium is satisfied. By increasing the mesh refinement, both DB formulations tend to the FB solution, although the DB/ae element model converges much faster than the one using classical displacement shape functions (e.g. the response with one $\mathrm{DB} / \mathrm{ae}$ element is superior to the one provided by two $\mathrm{DB} / \mathrm{c}$ elements).
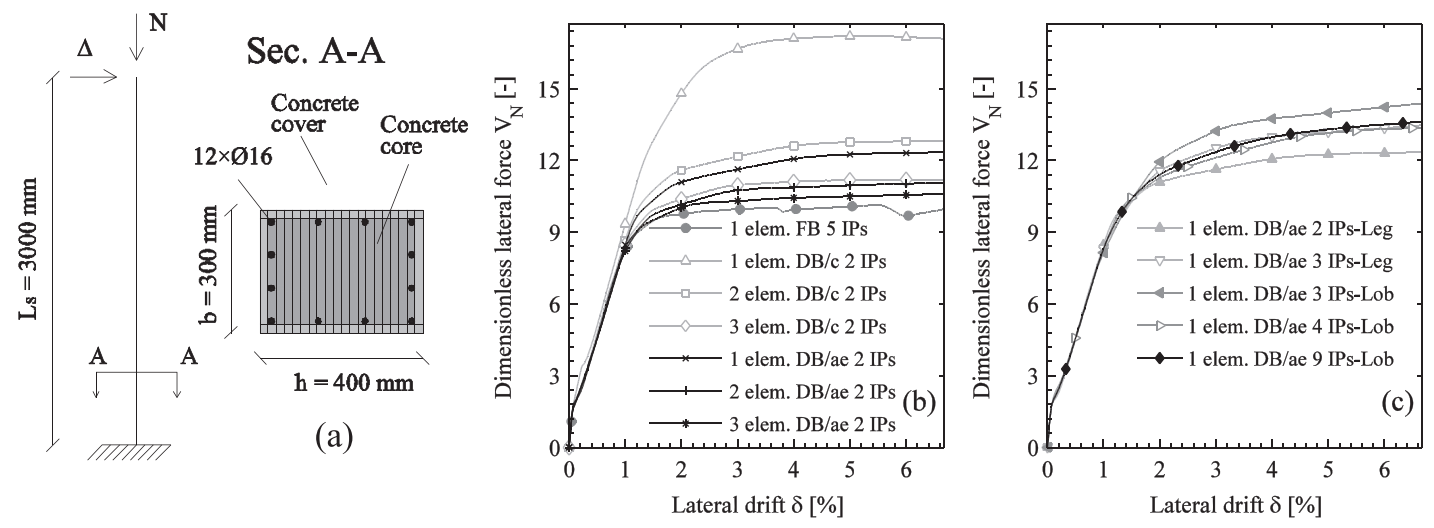

Figure 4. Application example: (a) structural representation and sectional discretization. Global-level response of DB/ae models: (b) influence of mesh refinement; (c) influence of element discretization. 


\section{AXIALLY EQUILIBRATED DISPLACEMENT-BASED ELEMENT FOR RC MEMBERS}

The impact of the number of IPs on the force-displacement response of the DB/ae element is shown in Figure 4(c). Different combinations of IPs and schemes (Gauss-Legendre/Gauss-Lobatto) were considered using a single FE to discretize the structural member. The figure shows that the pushover curves tend to a unique, objective solution-represented by the highly refined nine IPs GaussLobatto model - as the number of IPs increases. Further, a relatively low number of IPs, e.g. three Gauss-Legendre or four Gauss-Lobatto IPs, suffices to provide a satisfactory response. Even if two Gauss-Legendre or three Gauss-Lobatto are used, a relatively small numerical error of about $10 \%$ at peak response is observed for this example. Figure 4(c) also shows a further particular feature: while FB and DB formulations always provide an upper and lower bound for the strain energy, respectively, the proposed formulation does not offer a bound for this quantity. This relates to the exact verification of equilibrium, which is only achieved throughout the element for the axial force but not for the bending moment. Finally, the issue of localisation for softening sectional behaviour will not be addressed in the present document, although such pathology should occur for this formulation as it occurs for DB/c and FB approaches [10].

The local-level performance of models with a single DB/ae element (four Gauss-Lobatto IPs) and one $\mathrm{DB} / \mathrm{c}$ element (two Gauss-Legendre IPs) is compared in the following. Figure 5(a) starts by showing, for three different values of lateral drift, that curvature profiles are linear irrespective of the employed DB formulation. This is unsurprising because Hermitian shape functions are employed in both DB/ae and $\mathrm{DB} / \mathrm{c}$ approaches to define the element transversal displacement field. Note, however, that the curvature profiles of the DB/ae element are not quantitatively equal to those of a $\mathrm{DB} / \mathrm{c}$ element. In fact, although both $\mathrm{DB} / \mathrm{c}$ and $\mathrm{DB} / \mathrm{ae}$ elements are constrained to curvature linearity, the $\mathrm{DB} / \mathrm{ae}$ formulation verifies the principle of virtual work — as expressed by equation (9) — under a constant member axial force, while the $\mathrm{DB} / \mathrm{c}$ approach satisfies it by assuming a constant axial strain profile. This is shown in Figure 5(b), and, as expected, on account of the shifting of the neutral axis towards the compression side of the section, positive strain values (tension), which increase with drift demands, are observed. The figure also depicts the strain profiles for the DB/ae element, which evidence different values for distinct integration sections resulting from the iterative procedure to obtain a constant axial force along the column. The highest tensile average strain is recorded at the bottom IP, while at the element top, in correspondence of the inversion in sign of the curvature profile that takes place for large inelastic demands, small unrealistic tensile axial strains can be perceived. The constraint on the curvature profile is responsible for this effect. Overall, the DB/ae element better adheres to reality than the $\mathrm{DB} / \mathrm{c}$ element as the shifting of the neutral axis is expected to occur at the cantilever base where the bending moment is largest. Finally, Figure 5(c) shows the evolution of the axial force at distinct IPs with lateral drift. After an initial elastic phase, different IP axial forces occur in the DB/c element, which are symmetric around the value of the imposed axial load $\left(N^{I P_{j}}=N \pm \Delta N\right)$. This conservation of average equilibrium, which was noted elsewhere [10, 13], leads to an incorrect estimation of the flexural capacity of the structural member. This bias is not introduced by the DB/ae element, wherein the axial force in the four IPs is constant and equal to the external applied axial load.
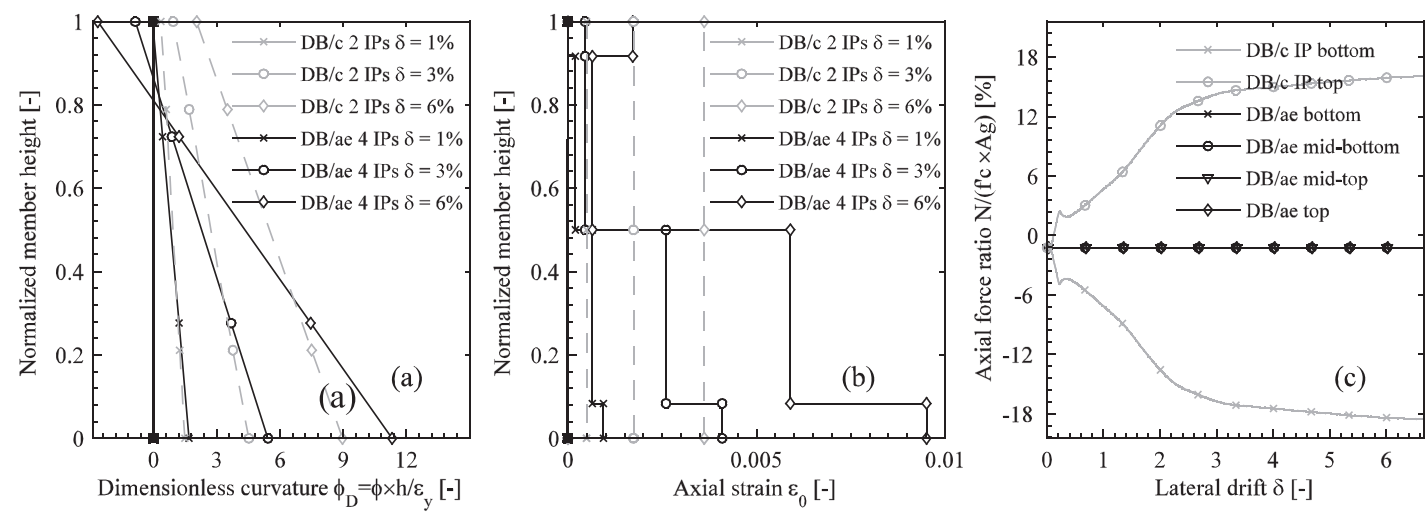

Figure 5. Comparison between DB/ae and DB/c element models at the local level: (a) curvature profiles; (b) vertical strain profiles; (c) axial force history in different IPs. 
The numerical performance of DB/ae and DB/c formulations is analysed in Figure 6(a) by comparing the number of NR iterations required to attain convergence. Two models providing similar accuracy at the global level response, as shown in Figure 4, were selected: a single DB/ae element with four Gauss-Lobatto IPs and two DB/c elements with two Gauss-Legendre IPs per element. Two to four iterations were typically necessary for both models, with the DB/ae converging faster on average. This observation is corroborated by the fact that a total of about 2700 and $3400 \mathrm{NR}$ iterations were needed for the DB/ae and DB/c model, respectively, see Figure 6(c). Although this could be expectable because only one DB/ae element is used, it is an encouraging indicator of the dependability of the element tangent stiffness matrix derived in equation (11). The DB/ae total and average number of internal axial equilibrium iterations per NR cycle at each load step are displayed in Figure 6(b) with black crosses and a grey line, respectively. An average of 2.5 iterations per NR cycle is required throughout the entire simulation, which represents an acceptable increase in computational time. However, as the classical DB formulations do not require this iterative procedure, they remain comparatively more performant time-wise, see Figure 6(c).

\section{VALIDATION EXAMPLES}

The accuracy of the DB/ae formulation is herein benchmarked against experimental data from two series of quasi-static cyclic tests on RC bridge piers (subsection 3.1) and RC walls (subsection 3.2). Models employing DB/c and FB elements are included in the comparison in order to point out the strengths and weaknesses of the proposed approach. The numerical results are compared against the experimental results with regard to global and local quantities.

\subsection{Tests on RC bridge piers}

The ability of the $\mathrm{DB} / \mathrm{ae}$ element to predict the nonlinear response of $\mathrm{RC}$ members is validated against a selection of quasi-static cyclic tests on circular RC bridge piers performed by Goodnight et al. [23]. The test units, which are listed in Table I, were selected to be representative of the largest possible spectrum of shear spans $L_{s} / D \in[4,8.67]$ and axial load ratios $N /\left(f_{c}^{\prime} \times A_{g}\right) \in[5 \%, 20 \%]$. The longitudinal and lateral reinforcement layout was common to all the specimens and consisted of 10 $\# 6$ rebars $(\approx \varnothing 19 \mathrm{~mm})$ and a \#3 $(\approx \varnothing 10 \mathrm{~mm})$ spiral at 2 inches $(\approx 50 \mathrm{~mm})$ pitch, corresponding to vertical and lateral reinforcement ratios that ranged between $\rho_{l} \in[1.6 \%, 1.7 \%]$ and $\rho_{w} \in[1 \%, 1.3 \%]$. A qualitative sketch of one of the test units, labelled as T9, is given in Figure 7(a).

All columns were subjected to a standard cyclic loading protocol with three cycles at each displacement amplitude. Target markers attached to the longitudinal rebars in the plastic hinge
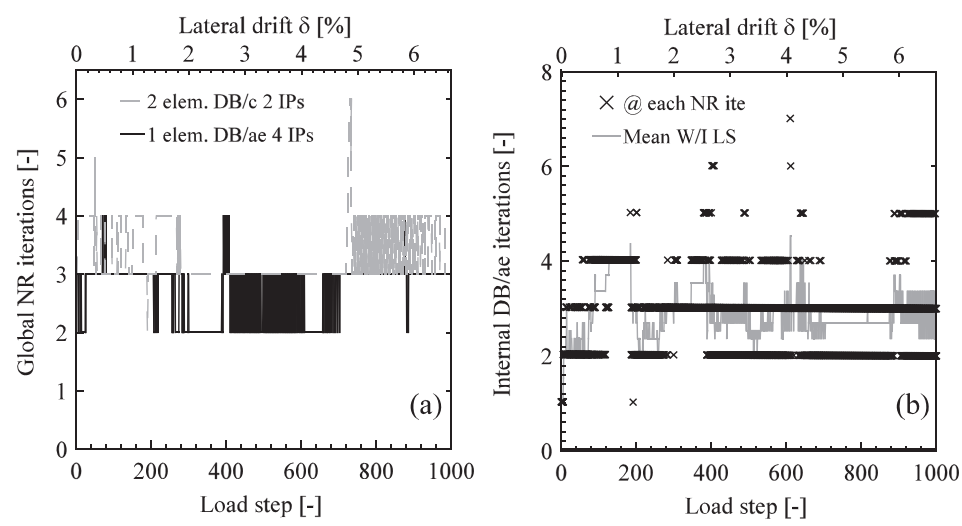

\begin{tabular}{c|cc} 
Formulation & DB/ae & DB/c \\
\hline No. of elements & 1 & 2 \\
IPs & 4 & 2 \\
Total NR ite. & 2700 & 3400 \\
Internal ite. avg. & 2.5 & {$[-]$} \\
Relative time & 1 & 0.65
\end{tabular}

Figure 6. Numerical performance of the DB/ae formulation: (a) DB/ae versus DB/c-Global NR iterations; (b) intra element iterations; (c) summary of results. 
Table I. Test matrix used for the validation of the proposed formulation (taken from [18]).

\begin{tabular}{|c|c|c|c|c|c|}
\hline Test & $D[\mathrm{~mm}]$ & $L_{s} / D[-]$ & Longitudinal reinforcement $\left(\rho_{l}\right)$ & Confining reinforcement $\left(\rho_{w}\right)$ & $N /\left(f^{\prime}{ }_{c} \times A_{g}\right)[-]$ \\
\hline T9 & 610 & 4 & $16 \varnothing 19(1.6 \%)$ & $\varnothing 10 @ 50$ mm (1\%) & $5.5 \%$ \\
\hline T19 & $457^{\mathrm{a}}$ & 5.33 & $16 \varnothing 19(1.7 \%)$ & $\varnothing 10 @ 50 \mathrm{~mm}(1.3 \%)$ & $10 \%$ \\
\hline $\mathrm{T} 20$ & $457^{\mathrm{a}}$ & 5.33 & $16 \varnothing 19(1.7 \%)$ & $\varnothing 10 @ 50 \mathrm{~mm}(1.3 \%)$ & $5 \%$ \\
\hline $\mathrm{T} 23$ & $457^{\mathrm{a}}$ & 8.67 & $16 \varnothing 19(1.7 \%)$ & $\varnothing 10 @ 50 \mathrm{~mm}(1.3 \%)$ & $5 \%$ \\
\hline $\mathrm{T} 24$ & $457^{\mathrm{a}}$ & 8.67 & $16 \varnothing 19(1.7 \%)$ & $\varnothing 10 @ 50 \mathrm{~mm}(1.3 \%)$ & $10 \%$ \\
\hline $\mathrm{T} 27$ & $610^{\mathrm{a}}$ & 4 & $16 \varnothing 19(1.6 \%)$ & Ø10@50mm (1\%) & $10 \%$ \\
\hline $\mathrm{T} 28$ & $457^{\mathrm{a}}$ & 5.33 & $16 \varnothing 19(1.7 \%)$ & $\varnothing 10 @ 50 \mathrm{~mm}(1.3 \%)$ & $15 \%$ \\
\hline T29 & $457^{\mathrm{a}}$ & 5.33 & $16 \varnothing 19(1.7 \%)$ & $\varnothing 10 @ 50 \mathrm{~mm}(1.3 \%)$ & $20 \%$ \\
\hline
\end{tabular}

$\mathrm{D}$, column diameter; $\mathrm{L}_{\mathrm{s}}$, shear span.

${ }^{\mathrm{a}}$ Nominal diameter, not accounting for the fact that cover concrete was not present in the instrumented region.

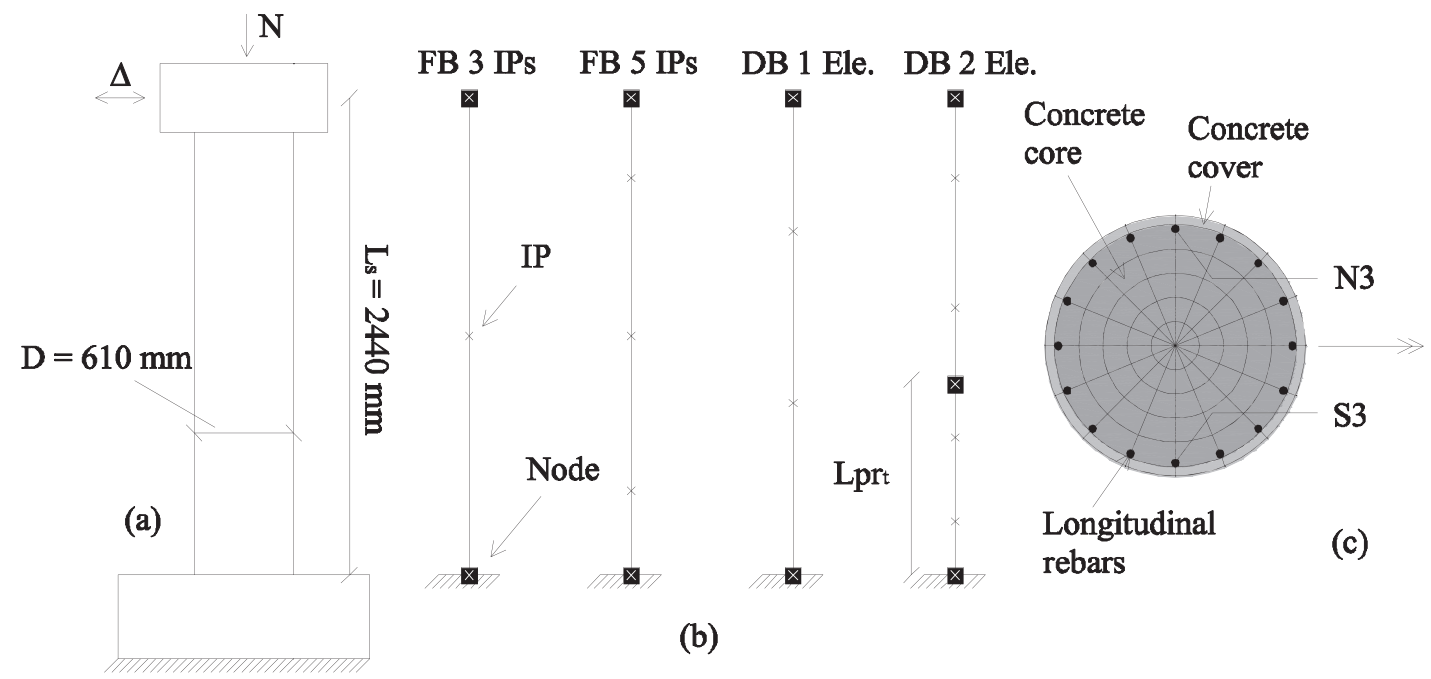

Figure 7. Test series by Goodnight et al. [23]: (a) sketch of test unit T9; (b) element formulation and discretization; (c) sectional discretization of test unit T9.

region tracked the displacements during the test and allowed to isolate the main deformation contributions to the total lateral displacement, namely base rotation and flexural displacements. Due to the relatively large shear span ratio of the considered test units, the impact of shear deformations was negligible.

The whole set of test units described in Table I was simulated with models employing DB/ae, DB/c and FB elements, following the four schemes depicted in Figure 7(b). Two models used a single FB element with three and five Gauss-Lobatto integration sections. The former represents the minimum number of IPs to simulate the linear response with a FB beam column element without underintegration [24]. However, not less than four IPs are recommended to simulate the nonlinear hardening response of structural members [25]. Despite such consideration, a discretization with three IPs was selected as it ensured for the studied specimens an influence length of the bottom IP similar to the measured extent of plasticity as discussed below. The fulfilment of this condition is recommended to optimize the agreement between the numerical results for FB elements and the experimental measurements at the local level [10]. For what concerns DB/c and DB/ae, models featuring one and two FEs per structural member were selected. Four Gauss-Lobatto IPs were used within each DB element for two reasons: (i) the Gauss-Lobatto quadrature rule allows to have an integration section at the element ends, which is useful if base curvatures are to be compared; and (ii) although the $\mathrm{DB} / \mathrm{c}$ formulation is insensitive to element discretization [13], this is not the case for DB/ae elements, which requires around four IPs as shown in Figure 4. For the cases where the structural member is discretized with two FEs, the length of the base element is selected as the 
upper bound of the measured extent of plasticity $-L p r_{t}$ in Figure 7(b), which is given by the following equation [26]:

$$
\operatorname{Lpr}_{t}=2 k L_{s}+0.75 D
$$

where $k=0.2 \times\left(f_{u} / f_{y}\right)-1<0.08$ is the factor accounting for the moment gradient as suggested by [2], $L_{s}$ is the shear span and $D$ is the column diameter. The same sectional discretization consisting of 80 confined concrete and 10 steel fibres is used to model the columns. They had no cover concrete in the plastic hinge region, and therefore no unconfined concrete fibres were defined. The exception was specimen T9, for which 16 unconfined concrete fibres had to be included as well for the sectional discretization as represented in Figure 7(c). The relationship proposed by Popovics-Mander [22] and Menegotto-Pinto [27] were used for the mechanical characterization of concrete and steel. The enhancement in concrete strength and strain at peak strength due to confinement were computed according to Mander's model [28]. The main material parameters used to characterize the concrete and steel stress-strain laws were derived from the actual material tests reported by Goodnight et al. [23], which differed for each tested specimen.

Table II reports the parameters used to model unit T9. Due to space constraints, the comparison between the numerical and experimental results shown in the next figures refers to this specimen alone.

The force-displacement $\mathrm{F}-\Delta$ response for all the models discussed above is depicted in Figure 8 and compared with the experimental measurements. A different graph is provided for each type of element formulation. Flexural displacements are reported on the bottom $x$-axis. These correspond to the total displacements of the numerical model; the experimental flexural displacements are computed by subtracting the displacement due to base rotation from the total displacement. The lateral displacement ductilities corresponding to the imposed demands of the cyclic loading protocol are shown in the top $x$-axis. The following observations can be made: (i) the FB models match satisfactorily the experimental data, with the model FB 3IPs slightly underestimating the actual response, which is typical for the bottom-up type of convergence of FB formulations [13]; (ii) both DB models using a single element overestimate the experimental $\mathrm{F}-\Delta$ curve, although the error

Table II. Main material parameters used in the numerical models of test T9.

\begin{tabular}{|c|c|c|c|c|c|c|c|c|}
\hline \multicolumn{5}{|c|}{ Concrete } & \multicolumn{4}{|c|}{ Reinforcing steel } \\
\hline$f^{\prime}{ }_{c}[\mathrm{MPa}]$ & $\varepsilon_{c}[\% o]$ & $E_{c}[\mathrm{GPa}]$ & ${f^{\prime}}_{c c}[\mathrm{MPa}]$ & $\varepsilon_{c c}[\% o]$ & $f_{y}[\mathrm{MPa}]$ & $f_{u}[\mathrm{MPa}]$ & $E_{s}[\mathrm{GPa}]$ & $b[\% o]$ \\
\hline 46.9 & 2 & 34.3 & 62.6 & 5.3 & 470 & 640 & 199.8 & 7.3 \\
\hline
\end{tabular}
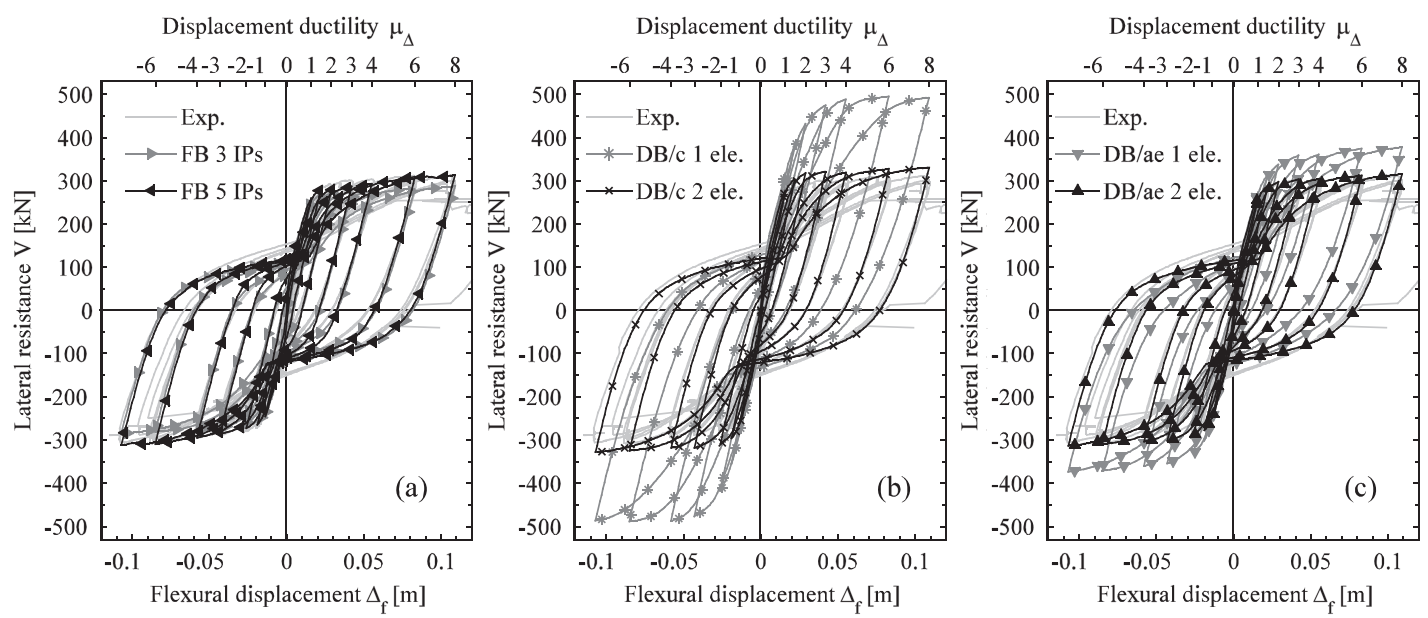

Figure 8. Experimental versus numerical force-displacement response for test T9: (a) FB, (b) DB/c and (c) DB/ae models. 

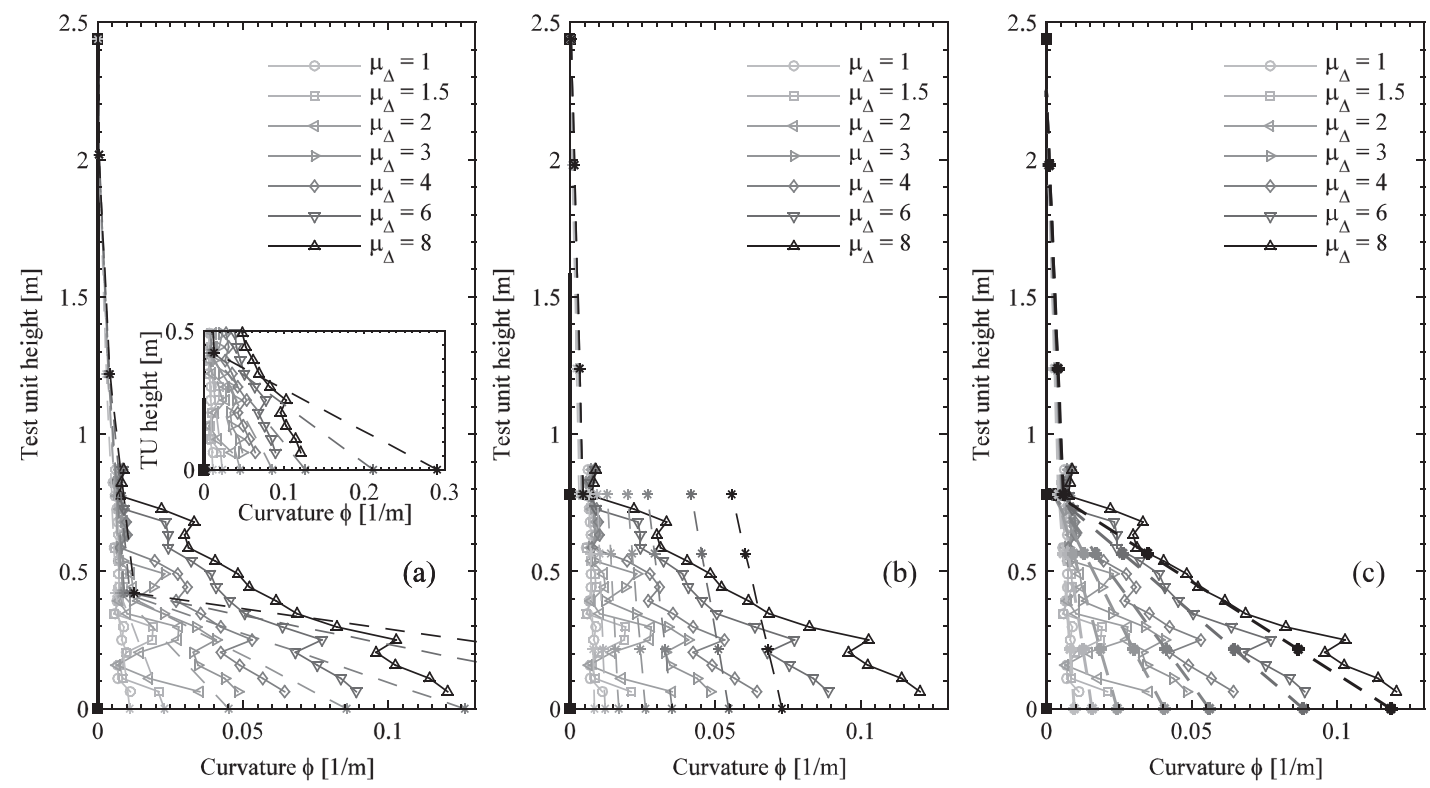

Figure 9. Experimental versus numerical curvature profiles for test T9 at positive ductility levels: (a) FB 5 IPs, (b) two-element DB/c and (c) two-element DB/ae models.

associated to the $\mathrm{DB} / \mathrm{ae}$ is sensibly lower; and (iii) a good match of the experimental $\mathrm{F}-\Delta$ curve is obtained by using two DB elements per member, both for the DB/c and the DB/ae approaches.

The DB/c and DB/ae with two elements per member and the FB element with five IPs (FB 5IP) are seen to perform better at the global level, which is the reason why they are chosen for the following comparison at the local level. The experimental and numerical curvature profiles for different levels of displacement ductility are shown in Figure 9. Taking into account the symmetry of the system, only the curvature profiles in the positive direction of loading were analysed. Increasing displacement ductility levels from yielding $\left(\mu_{\Delta}=1\right)$ up to $\mu_{\Delta}=8$ were considered. Within each element, the obtained curvatures at the successive IPs are connected with a straight line. For DB elements, where the linearity of curvatures is imposed, this representation of the curvature profiles is exact; for FB elements, it corresponds instead to a slight overestimation of the real curvature distribution along the element. The better match of the DB/ae formulation is apparent: (i) the model FB 5IPs tends to overestimate the base curvature, and markedly so for large drift levels. As an example, the relative error ${ }^{1}$ corresponding to the base curvature $\left(\eta_{\phi b}\right)$ for a ductility demand $\mu_{\Delta}=8$ is approximately $110 \%$; (ii) the opposite trend applies to the $\mathrm{DB} / \mathrm{c}$ model, which underestimates the experimental base curvature $\left(\eta_{\phi b}=50 \%\right.$ for $\left.\mu_{\Delta}=8\right)$. Moreover, when such elements are employed, it is worthy to notice how the numerical curvature profiles are not continuous along the member length, which originates from the non-strict verification of equilibrium along each FE; (iii) the match between observed and calculated curvature profiles is remarkably improved when DB/ae are used, showing a relative error for the base curvature at $\mu_{\Delta}=8$ smaller than $5 \%$. The agreement between analytical and experimental base curvature appears to decrease with the attained ductility level, with a maximum relative error of $35 \%$ for $\mu_{\Delta}=2$. This can be attributed to the use of a constant bottom element length, which does not reflect the experimentally observed decrease on the extent of plasticity with ductility demand [18]. Finally, observe that the strict verification of axial equilibrium almost completely eliminates the discontinuity in curvature between the bottom and upper element.

The vertical strains are depicted in Figures 10 and 11 for the two outmost rebars in tension (N3) and compression (S3), which are indicated in Figure 7(c). Once again, for both cases, the DB/ae provides improved strain predictions with respect to $\mathrm{DB} / \mathrm{c}$ and FB elements models, which tend to underestimate and overestimate, respectively, the maximum experimental strains. If base strains are averaged over all ductility levels, the following relative errors are obtained: $\eta_{\varepsilon b}^{N 3}=75 \%$ and $\eta_{\varepsilon b}^{S 3}=65 \%$ for FB, $\eta_{\varepsilon b}^{N 3}=44 \%$

$\overline{{ }^{1} \text { Relative error: } \eta_{v}}=\left|1-\frac{v_{\text {exp }}}{v_{\text {num }}}\right|$. 

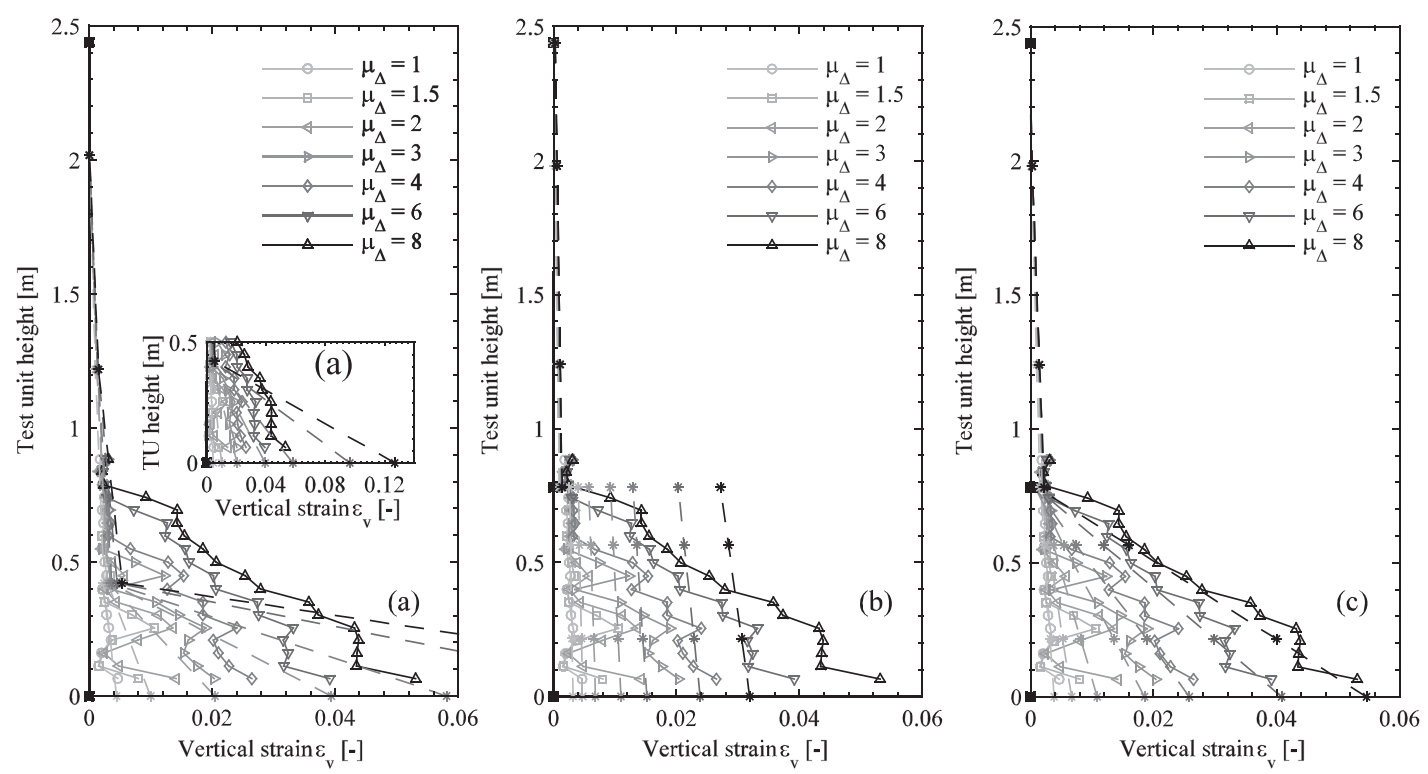

Figure 10. Experimental versus numerical vertical strain profiles of rebar N3 for test T9 at positive ductility levels: (a) FB 5 IPs, (b) two-element DB/c and (c) two-element DB/ae models.
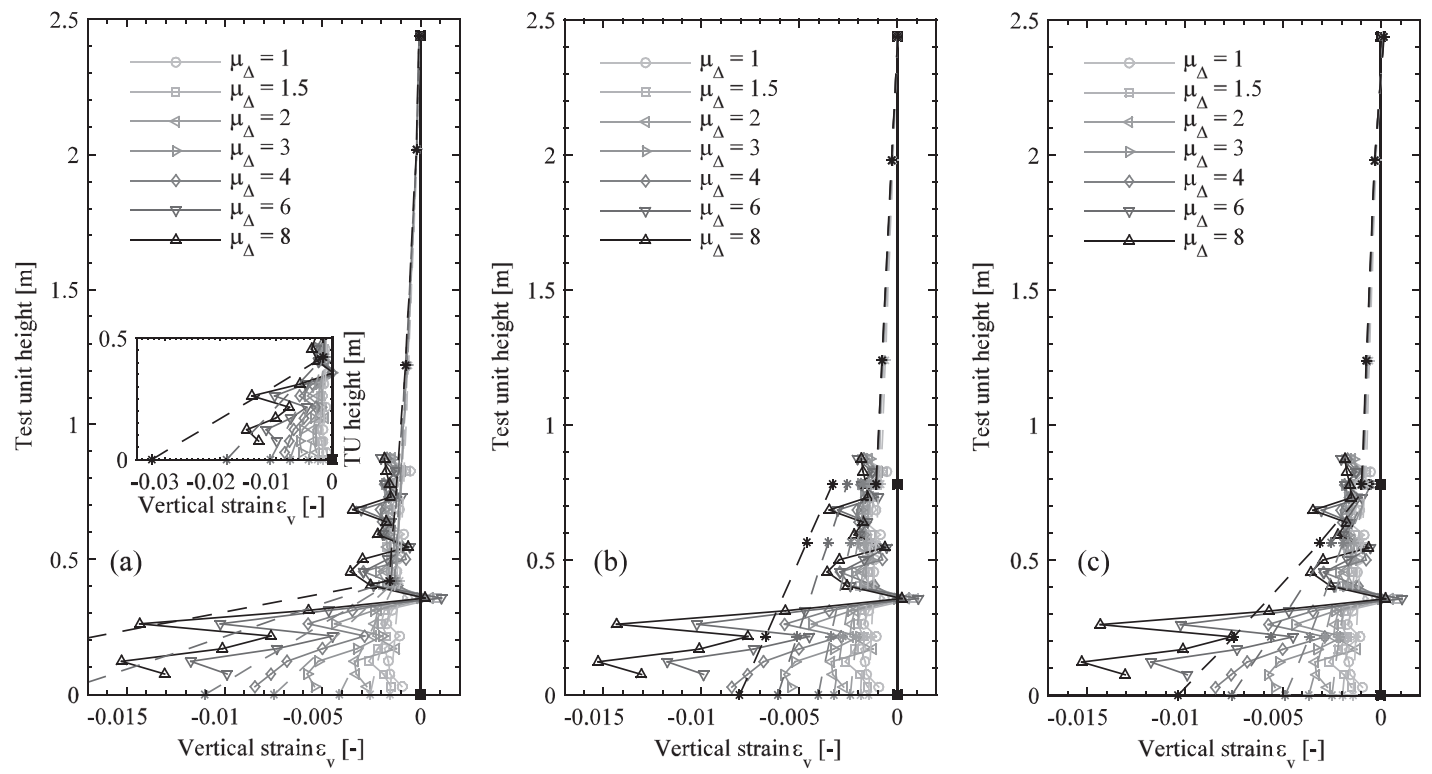

Figure 11. Experimental versus numerical vertical strain profiles of rebar S3 for test $\mathrm{T} 9$ at positive ductility levels: (a) FB 5 IPs, (b) two-element DB/c and (c) two-element DB/ae models.

and $\eta_{\varepsilon b}^{S 3}=37 \%$ for $\mathrm{DB} / \mathrm{c}, \eta_{\varepsilon b}^{N 3}=9 \%$ and $\eta_{\varepsilon b}^{S 3}=25 \%$ for DB/ae models. The errors for DB/ae reduce to $\eta_{\varepsilon b}^{N 3}$ $=2 \%$ and $\eta_{\varepsilon b}^{S 3}=18 \%$ if only large ductility levels are considered $\left(\mu_{\Delta}=6\right.$ and $\left.\mu_{\Delta}=8\right)$. The discrepancy between numerical and experimental strains obtained for the rebar in compression with respect to the one in tension is due to the following reasons: (i) compression strain profiles are not as linear as their counterpart in tension as they are more influenced by phenomena occurring at the micro-level; (ii) the height at which compression strains deviate from linearity is smaller than the extent of plasticity $\left(L p r_{t}\right)$ used to discretize the structural member. A different (shorter) length of the bottom $\mathrm{DB} / \mathrm{ae}$ element would therefore be needed to improve the simulation of compressive strain profiles. For this reason, in the framework of plastic hinge models, Goodnight et al. [26] proposed a different 


\section{AXIALLY EQUILIBRATED DISPLACEMENT-BASED ELEMENT FOR RC MEMBERS}
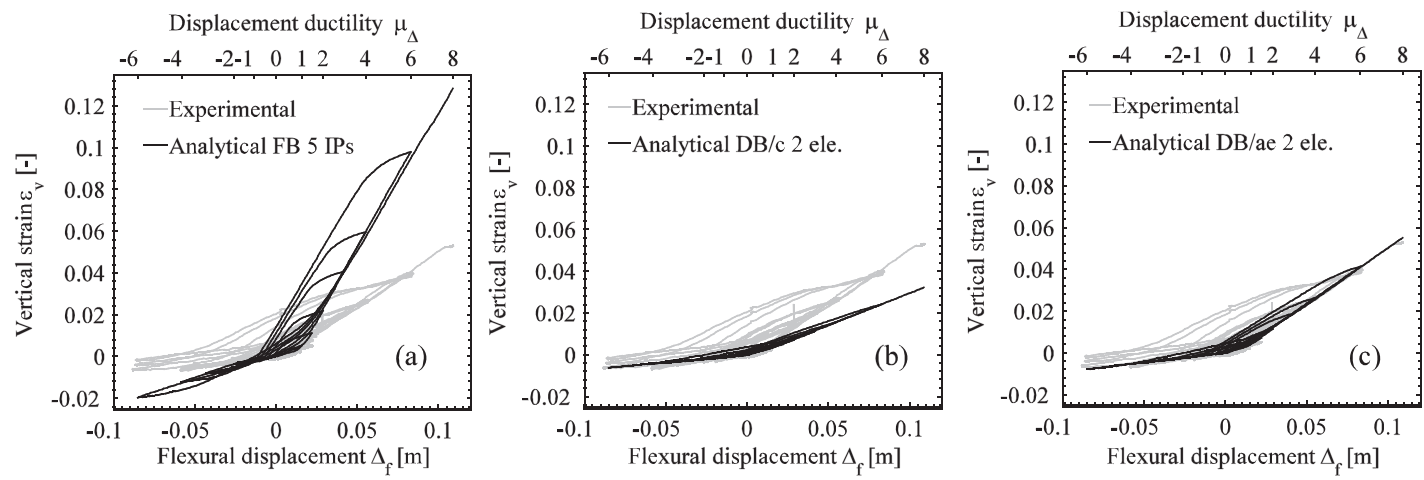

Figure 12. Experimental versus numerical vertical strain history of rebar N3 measured at the base section of test T9 for positive ductility levels: (a) FB 5 IPs, (b) two-element DB/c and (c) two-element DB/ae models.

plastic hinge length $\operatorname{Lpr}_{c}$ (to be used in conjunction with a bilinear curvature profile) to reliably evaluate limit state displacements based on compression strains. Nevertheless, changing the length of the base DB/ae element would then inevitably lead to a poorer prediction of both the curvatures and tensile strains. The latter have a more clear influence on the measured curvatures because, for the same level of top displacement, they are in absolute value considerably larger than compression strains.

As a further local level investigation, the comparison between the experimental vertical strains of rebar N3 monitored at the bottom of the RC column and the numerical ones (measured at the bottom IP) is shown in Figure 12. Again, the DB/ae model offers the best agreement between simulation and test data, especially regarding the tensile peak strain levels. An underestimation of

Table III. Numerical error in the calculation of the base curvature for all the models and selected test units tested by Goodnight et al. [23] at different ductility levels.

\begin{tabular}{|c|c|c|c|c|c|c|c|}
\hline \multirow[b]{2}{*}{ Test } & \multirow{2}{*}{$\begin{array}{c}\text { Ductility } \\
\text { level } \\
\left(\mu_{\Delta}\right)\end{array}$} & \multicolumn{4}{|c|}{ Base curvature average relative error: $\boldsymbol{\eta}_{\phi \boldsymbol{b}}^{a v g}=\frac{1}{2}$} & $\left|1-\frac{\left(\phi_{b, a n}^{+}\right)}{\phi_{b, e x p}^{+}}\right|$ & $\left.-\left|1-\frac{\left(\phi_{b, a n}^{-}\right)}{\phi_{b, \text { exp }}^{-}}\right|\right)$ \\
\hline & & FB 3IPs & FB 5IPs & DB/c 1 ele. & DB/c 2 ele. & DB/ae 1 ele. & DB/ae 2 ele. \\
\hline \multirow[t]{3}{*}{ T9 } & $\mu_{\Delta}= \pm 2$ & $43.6 \%$ & $18.3 \%$ & $65.1 \%$ & $55.5 \%$ & $59.9 \%$ & $35.6 \%$ \\
\hline & $\mu_{\Delta}= \pm 4$ & $28.3 \%$ & $92.7 \%$ & $61.2 \%$ & $47.2 \%$ & $54.6 \%$ & $15.6 \%$ \\
\hline & $\mu_{\Delta}= \pm 6$ & $19.5 \%$ & $124.8 \%$ & $58.1 \%$ & $41.8 \%$ & $50.0 \%$ & $5.8 \%$ \\
\hline \multirow[t]{3}{*}{ T19 } & $\mu_{\Delta}= \pm 2$ & $45.2 \%$ & $\mathbf{5 . 9} \%$ & $64.6 \%$ & $53.9 \%$ & $61.4 \%$ & $35.1 \%$ \\
\hline & $\mu_{\Delta}= \pm 4$ & $37.6 \%$ & $53.9 \%$ & $64.1 \%$ & $49.7 \%$ & $60.2 \%$ & $22.5 \%$ \\
\hline & $\mu_{\Delta}= \pm 6$ & $32.7 \%$ & $68.5 \%$ & $62.3 \%$ & $46.1 \%$ & $57.7 \%$ & $16.1 \%$ \\
\hline \multirow[t]{3}{*}{$\mathrm{T} 20$} & $\mu_{\Delta}= \pm 2$ & $49.0 \%$ & $6.3 \%$ & $67.4 \%$ & $58.0 \%$ & $63.6 \%$ & $38.5 \%$ \\
\hline & $\mu_{\Delta}= \pm 4$ & $33.6 \%$ & $58.7 \%$ & $62.6 \%$ & $48.1 \%$ & $57.5 \%$ & $17.6 \%$ \\
\hline & $\mu_{\Delta}= \pm 6$ & $33.1 \%$ & $46.8 \%$ & $62.9 \%$ & $48.4 \%$ & $57.7 \%$ & $16.8 \%$ \\
\hline \multirow[t]{3}{*}{$\mathrm{T} 23$} & $\mu_{\Delta}= \pm 2$ & $52.1 \%$ & $5.6 \%$ & $69.4 \%$ & $56.0 \%$ & $65.8 \%$ & $34.9 \%$ \\
\hline & $\mu_{\Delta}= \pm 4$ & $39.0 \%$ & $45.9 \%$ & $65.5 \%$ & $45.0 \%$ & $60.9 \%$ & $11.2 \%$ \\
\hline & $\mu_{\Delta}= \pm 6$ & $35.6 \%$ & $26.2 \%$ & $64.7 \%$ & $42.7 \%$ & $59.6 \%$ & $4.2 \%$ \\
\hline \multirow[t]{3}{*}{$\mathrm{T} 24$} & $\mu_{\Delta}= \pm 2$ & $51.0 \%$ & $5.4 \%$ & $68.2 \%$ & $54.2 \%$ & $65.2 \%$ & $34.8 \%$ \\
\hline & $\mu_{\Delta}= \pm 4$ & $40.1 \%$ & $47.5 \%$ & $65.5 \%$ & $44.5 \%$ & $61.7 \%$ & $12.9 \%$ \\
\hline & $\mu_{\Delta}= \pm 6$ & $38.1 \%$ & $55.9 \%$ & $65.4 \%$ & $42.4 \%$ & $61.1 \%$ & $8.4 \%$ \\
\hline \multirow[t]{3}{*}{$\mathrm{T} 27$} & $\mu_{\Delta}= \pm 2$ & $32.4 \%$ & $44.2 \%$ & $58.1 \%$ & $47.3 \%$ & $53.9 \%$ & $23.4 \%$ \\
\hline & $\mu_{\Delta}= \pm 4$ & $17.7 \%$ & $111.6 \%$ & $53.8 \%$ & $38.9 \%$ & $48.3 \%$ & $5.9 \%$ \\
\hline & $\mu_{\Delta}= \pm 6$ & $16.5 \%$ & $119.7 \%$ & $54.2 \%$ & $38.3 \%$ & $47.9 \%$ & $\mathbf{5 . 0} \%$ \\
\hline \multirow[t]{3}{*}{$\mathrm{T} 28$} & $\mu_{\Delta}= \pm 2$ & $35.0 \%$ & $34.0 \%$ & $58.5 \%$ & $45.3 \%$ & $55.7 \%$ & $21.9 \%$ \\
\hline & $\mu_{\Delta}= \pm 4$ & $30.8 \%$ & $79.9 \%$ & $59.8 \%$ & $44.1 \%$ & $56.5 \%$ & $13.7 \%$ \\
\hline & $\mu_{\Delta}= \pm 6$ & $30.0 \%$ & $88.5 \%$ & $60.3 \%$ & $43.4 \%$ & $56.4 \%$ & $12.3 \%$ \\
\hline \multirow[t]{3}{*}{$\mathrm{T} 29$} & $\mu_{\Delta}= \pm 2$ & $37.0 \%$ & $31.7 \%$ & $59.2 \%$ & $45.8 \%$ & $57.4 \%$ & $24.8 \%$ \\
\hline & $\mu_{\Delta}= \pm 4$ & $31.6 \%$ & $78.7 \%$ & $59.3 \%$ & $43.5 \%$ & $57.2 \%$ & $14.7 \%$ \\
\hline & $\mu_{\Delta}= \pm 6$ & $30.2 \%$ & $90.8 \%$ & $59.3 \%$ & $42.3 \%$ & $56.7 \%$ & $12.5 \%$ \\
\hline
\end{tabular}

Note: minimum values of $\boldsymbol{\eta}_{\phi \boldsymbol{b}}^{a v g}$ for each test unit and ductility level are displayed in bold 
the residual strains at zero displacement level is instead common to all three models, indicating that the accumulation of plastic reinforcement strains over multiple cycles is not well captured. However, this issue is not directly related to the element formulation and could arguably be addressed with more advanced steel and concrete constitutive relationships for cyclic response.

Finally, Table III compares the experimental base curvatures of the entire dataset units with all the employed models for three distinct values of displacement ductility $\left(\mu_{\Delta}=2, \mu_{\Delta}=4\right.$ and $\left.\mu_{\Delta}=6\right)$. The comparison is made in terms of average relative error $\left(\eta_{\phi b}^{a v g}\right)$, which is defined as the mean of the relative base curvatures errors for the positive and negative directions of loading. For each test unit and ductility level, the minimum value of $\eta_{\phi b}^{a v g}$ is highlighted in bold in Table III. For most cases, the model composed of two DB/ae elements per structural member provides the best simulation of base curvatures. The $\eta_{\phi b}^{a v g}$ predicted by such model results generally smaller than $20 \%$ and tends to decrease for increasing inelastic demands. Values of $\eta_{\phi b}^{a v g}$ larger than $30 \%$ are obtained only for $\mu_{4}=2$ in tests T9, T19, T20, T23 and T24, in which cases the model FB 5 IPs leads to the best predictions. For larger values of $\mu_{\Delta}$, the experimental base curvatures are consistently and largely overestimated by the model FB 5IPs, as confirmed by Figure 9 .

\subsection{Tests on RC structural walls}

Five out of the six RC walls (labelled WSH1 to WSH6) from the experimental campaign carried out by Dazio et al. [29] are used in this subsection for validation purposes. Wall WSH1 was disregarded due to the poor ductility properties of the longitudinal reinforcement which led the specimen to fail at a low level of inelasticity. Moreover, only manual measurements were employed to evaluate local level quantities of WSH1, which were judged less reliable than those of all the other tests where hard wired instruments were used.

The main geometrical and loading characteristics of the test specimens are shown in Table IV. A constant vertical load was applied at the top of the specimens, which were then subjected to a standard cyclic loading protocol [30]. The test units differed mainly with regard to the layout and content of both longitudinal and horizontal reinforcement, as well as to the applied axial load ratio.

Local deformations were obtained from linear variable differential transformers (LVDTs) and Demec measurements, allowing to isolate the different contributions to the total lateral displacement, namely due to flexure, base rotation and shear. The latter played a non-negligible role (up to a maximum of around $10 \%$ of the total deformation) due to the small shear span ratios and thus could not be disregarded. In the framework of EB beam theory, shear deformations are not considered, and therefore, in order to compare consistently numerical and experimental results, their contribution had to be removed from the total lateral displacement. However, this does not represent a limitation to the present validation example because (i) the proposed formulation can be extended to a more general one accounting for shear deformations, e.g. Timoshenko beam theory; and (ii) shear deformation can be included separately as a ratio of the flexural displacement [2, 31-33].

The same models described in the previous subsection 3.1-i.e. FB 5IPs, FB 3IPs, one and twoelement $\mathrm{DB} / \mathrm{c}$, one and two-element $\mathrm{DB} / \mathrm{ae}$ - are employed to simulate the set of chosen $\mathrm{RC}$ walls.

Table IV. Main properties of test units by Dazio et al. [29] used for validation of the DB/ae formulation.

\begin{tabular}{lccccccccc}
\hline & $L_{S}$ & $h$ & $L_{s} / h$ & $t$ & $N /\left(f^{\prime}{ }_{c} \times A_{g}\right)$ & $\rho_{l, \text { bound }}$ & $\rho_{l, \text { web }}$ & $\rho_{h}$ & Conf. BE \\
\cline { 2 - 10 } Test unit & {$[\mathrm{mm}]$} & {$[\mathrm{mm}]$} & {$[-]$} & {$[\mathrm{mm}]$} & {$[\%]$} & {$[\%]$} & {$[\%]$} & {$[\%]$} & {$[-]$} \\
\hline WSH2 & 4560 & 2000 & 2.28 & 150 & 5.7 & 1.32 & 0.30 & 0.25 & $\checkmark$ \\
WSH3 & 4560 & 2000 & 2.28 & 150 & 5.8 & 1.54 & 0.54 & 0.25 & $\checkmark$ \\
WSH4 & 4560 & 2000 & 2.28 & 150 & 5.7 & 1.54 & 0.54 & 0.25 & $x$ \\
WSH5 & 4560 & 2000 & 2.28 & 150 & 12.8 & 0.67 & 0.24 & 0.25 & $\checkmark$ \\
WSH6 & 4520 & 2000 & 2.20 & 150 & 10.8 & 1.54 & 0.54 & 0.25 & $\checkmark$ \\
\hline
\end{tabular}

$L_{s}$, shear span; $h$, wall length; $t$, wall thickness; $N /\left(f^{\prime} c \times A g\right)$, axial load ratio; $\rho_{l, \text { bound }}$, boundary elements longitudinal reinforcement ratio; $\rho_{l, w e b}$, web longitudinal reinforcement ratio; $\rho_{h}$, horizontal reinforcement ratio; Conf. BE, additional confining and stabilizing reinforcement in the boundary elements (hoops and ties). 


\section{AXIALLY EQUILIBRATED DISPLACEMENT-BASED ELEMENT FOR RC MEMBERS}

Due to space constraints, only test unit WSH6 is used in the following paragraphs to show and discuss the comparison between numerical and experimental results, both at the global and local levels. The summary of the response of the entire set of RC walls is included in a table provided at the end of the present subsection.

The geometry and cross-sectional reinforcement layout of wall WSH6 are depicted in Figure 13(a) and (b). Well-confined boundary elements were present to increase the flexural capacity of the structural member. The longitudinal reinforcement consisted of $22 \varnothing 8-\mathrm{mm}$ and $6 \varnothing 12-\mathrm{mm}$ bars for the web $\left(\rho_{w}=0.54 \%\right)$ and boundary elements $\left(\rho_{b}=1.54 \%\right)$. The shear reinforcement was composed of $\varnothing 6-\mathrm{mm}$ bars spaced $150 \mathrm{~mm}$ while $\varnothing 6-\mathrm{mm}$ and $\varnothing 4.2-\mathrm{mm}$ hoops at $50 \mathrm{~mm}$ were employed to properly confine the wall edges. The fibre sectional discretization used in the numerical models is displayed in Figure 13(c). As in the previous subsection, the material models proposed by PopovicsMander [22] and Menegotto-Pinto [27] were adopted to characterize the concrete and steel stressstrain laws. Different confinement factors were computed according to the model by Mander et al. [28] for the core concrete in the web and in the boundary elements. The main material parameters are given in Table $\mathrm{V}$. When two elements were employed to discretize the structural member $(\mathrm{DB} / \mathrm{c}$ and $\mathrm{DB} / \mathrm{ae}$ ), the length of the one at the bottom was taken as the height of the plastic zone $L_{p z}$, which is defined by Dazio et al. [29] as the height at which the plastic curvature profile is equal to the yield curvature.

The experimental and numerical force-displacement responses of wall WSH6 are contrasted in Figure 14 . On the bottom $x$-axis, flexural displacements $\Delta_{f}$ were calculated by subtracting the displacements due to base rotation and shear deformations from the total lateral displacements. Displacement ductility $\mu_{\Delta}$ and lateral resisting force $V$ are instead represented on the top $x$-axis and vertical $y$-axis. The two DB models using two elements per structural member satisfactorily reproduce the experimental results. Namely, the force capacity at all displacement reversals is adequately captured, with relative errors $\eta_{V}<5 \%$. FB models are slightly less accurate, underestimating the resisting force at loading reversals ( $\eta_{V}$ up to $12 \%$ ). As expected, the DB models using a single FE overestimate the strength capacity of the structural member, although this effect is significantly less pronounced for the DB/ae model.

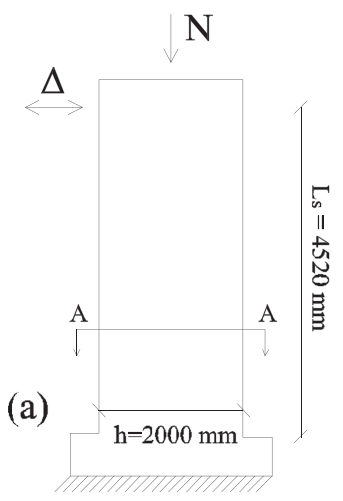

Figure 13. Test unit WSH6:

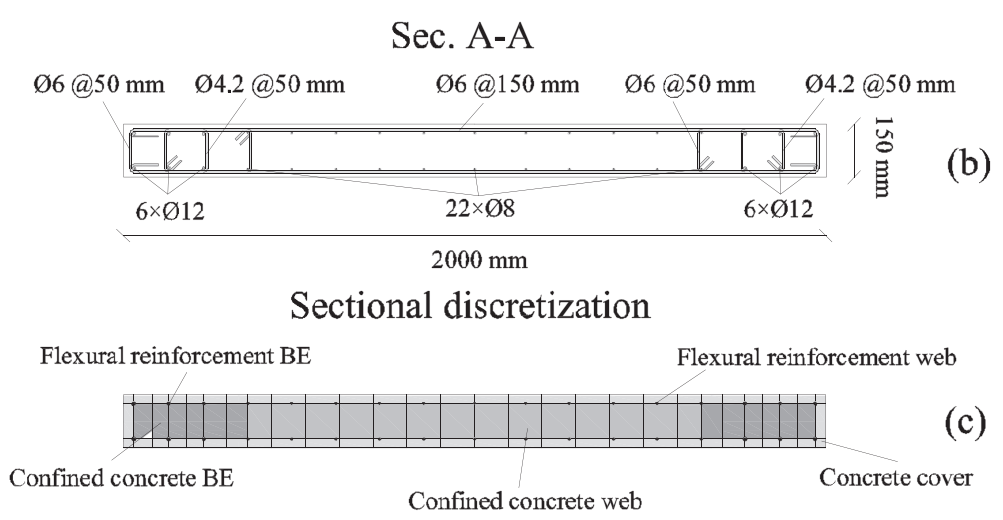

(a) sketch of geometry and applied loading, (b) reinforcement layout and (c) sectional discretization.

Table V. Main material parameters used in the numerical models of test WSH6, derived from the properties reported by Dazio et al. [29].

\begin{tabular}{|c|c|c|c|c|c|c|c|c|c|c|}
\hline & \multicolumn{5}{|c|}{ Concrete } & & \multicolumn{4}{|c|}{ Reinforcing steel } \\
\hline & $f_{c}^{\prime}{ }_{c}[\mathrm{MPa}]$ & $\varepsilon_{c}[\% o]$ & $E_{c}[\mathrm{GPa}]$ & $f^{\prime}{ }_{c c}[\mathrm{MPa}]$ & $\varepsilon_{c c}[\% o]$ & & $f_{y}[\mathrm{MPa}]$ & $f_{u}[\mathrm{MPa}]$ & $E_{s}[\mathrm{GPa}]$ & $b[\%]$ \\
\hline Web & 45.6 & 2 & 36.9 & 48.4 & 2.6 & $\varnothing 8 \mathrm{~mm}$ & 576 & 675 & 200 & 9.3 \\
\hline Boundary & 45.6 & 2 & 36.9 & 53.5 & 5.1 & $\varnothing 12 \mathrm{~mm}$ & 583 & 714 & 200 & 8.4 \\
\hline
\end{tabular}



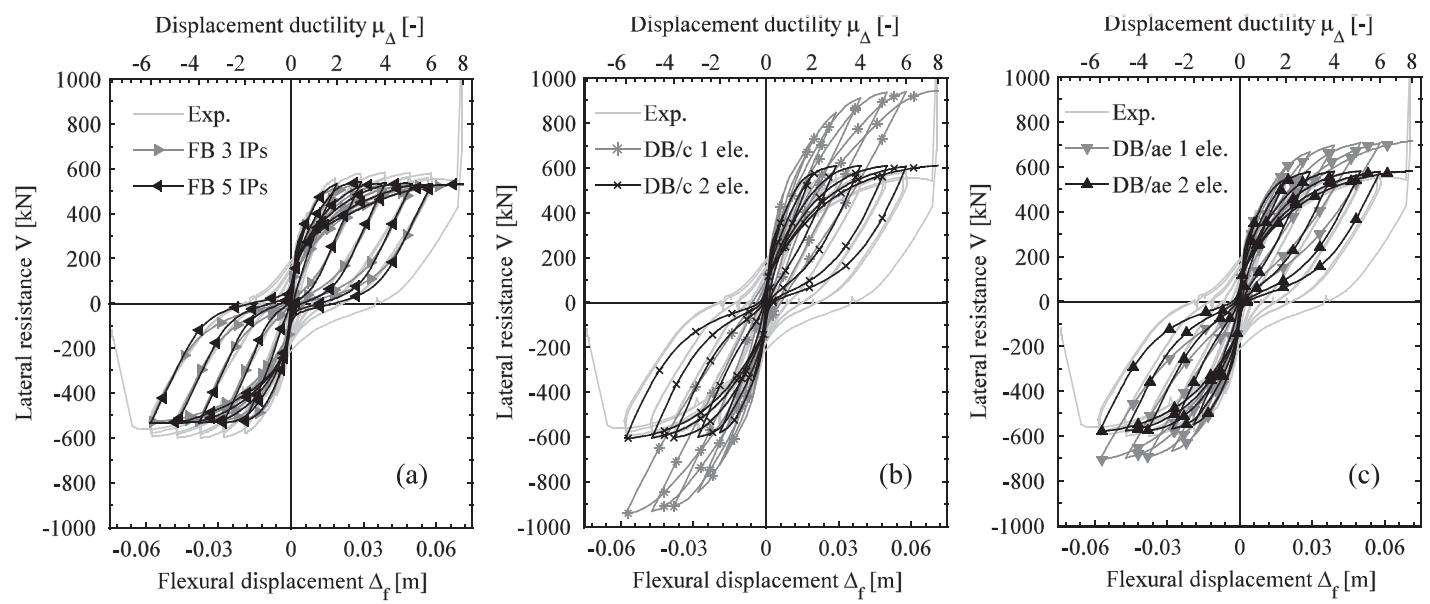

Figure 14. Experimental versus numerical force-displacement response for test WSH6: (a) FB, (b) DB/c and (c) DB/ae models.

At the local scale of analysis, the numerical versus the experimental curvature and strain profiles of corner rebars are displayed in Figures 15 and 16. Only the models yielding the best match at the global level are included in the comparison, i.e. FB 5IPs, DB/c and DB/ae with two elements per structural member. Several displacement ductility demands are considered, each one corresponding to a point of reversal in the hysteretic force-displacement response of Figure 14. Due to the symmetry of both specimen cross section and loading protocol, only curvature and strain profiles relative to positive top displacements are shown.

The DB/ae element model best matches the experimental curvatures, with $\eta_{\phi b}<10 \%$ for all ductility levels. For large values of $\mu_{\Delta}$, the base curvatures are greatly underestimated by the $\mathrm{DB} / \mathrm{c}$ model (e.g. $\eta_{\phi b}>40 \%$ for $\mu_{\Delta}=8$ ) and overestimated by the FB 5IPs model (e.g. $\eta_{\phi b}>120 \%$ for $\mu_{\Delta}=8$ ). Similar comments apply regarding the tensile strain profiles of Figure 16. Strain profiles in compression are instead best captured by the model FB 5IPs. Although the DB/ae model performs slightly better than the $\mathrm{DB} / \mathrm{c}$, both underestimate the recorded maximum compressive strain. As discussed in subsection 3.1, this is a direct consequence of the assumed FE discretization of the structural
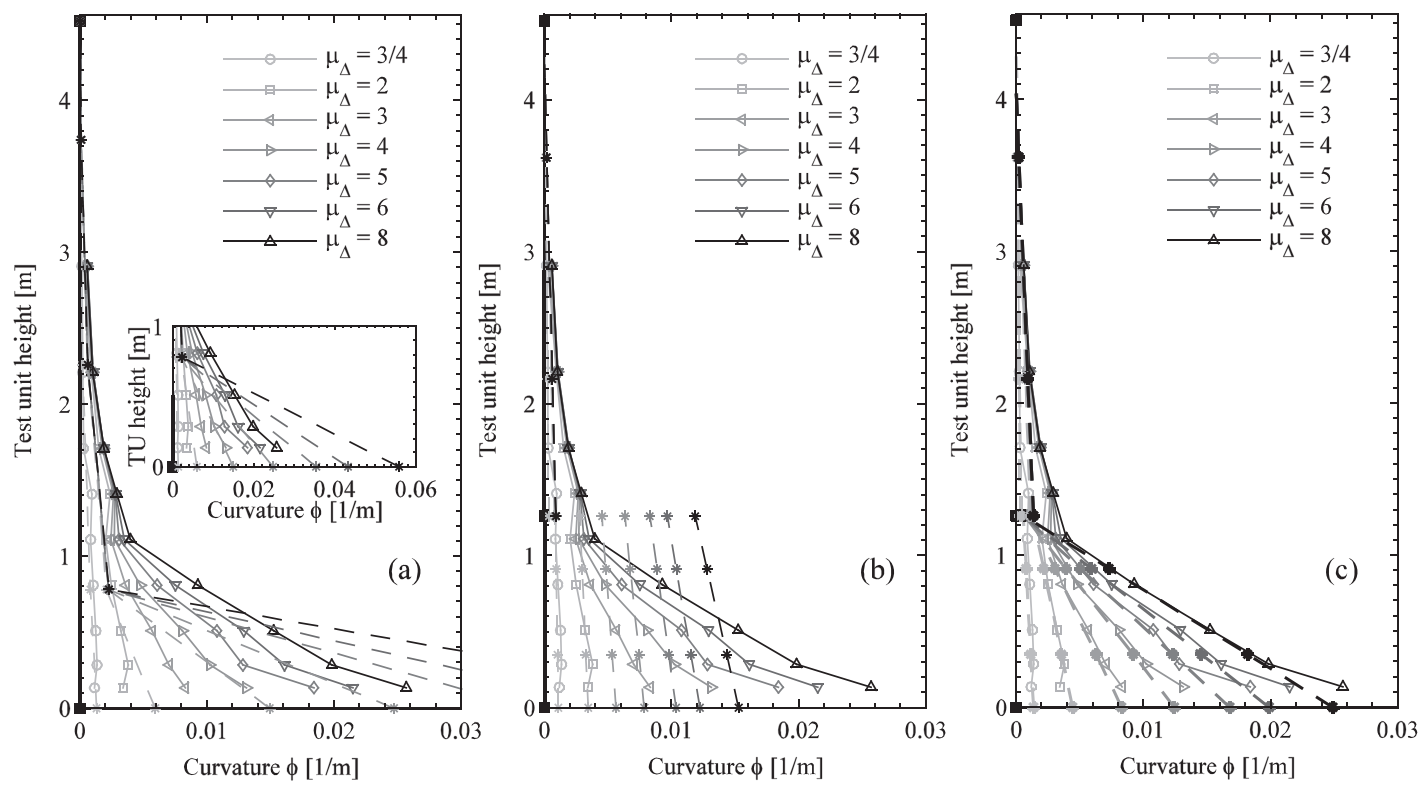

Figure 15. Experimental versus numerical curvature profiles for test WSH6 at positive ductility levels: (a) FB 5 IPs, (b) two-element DB/c and (c) two-element DB/ae models. 

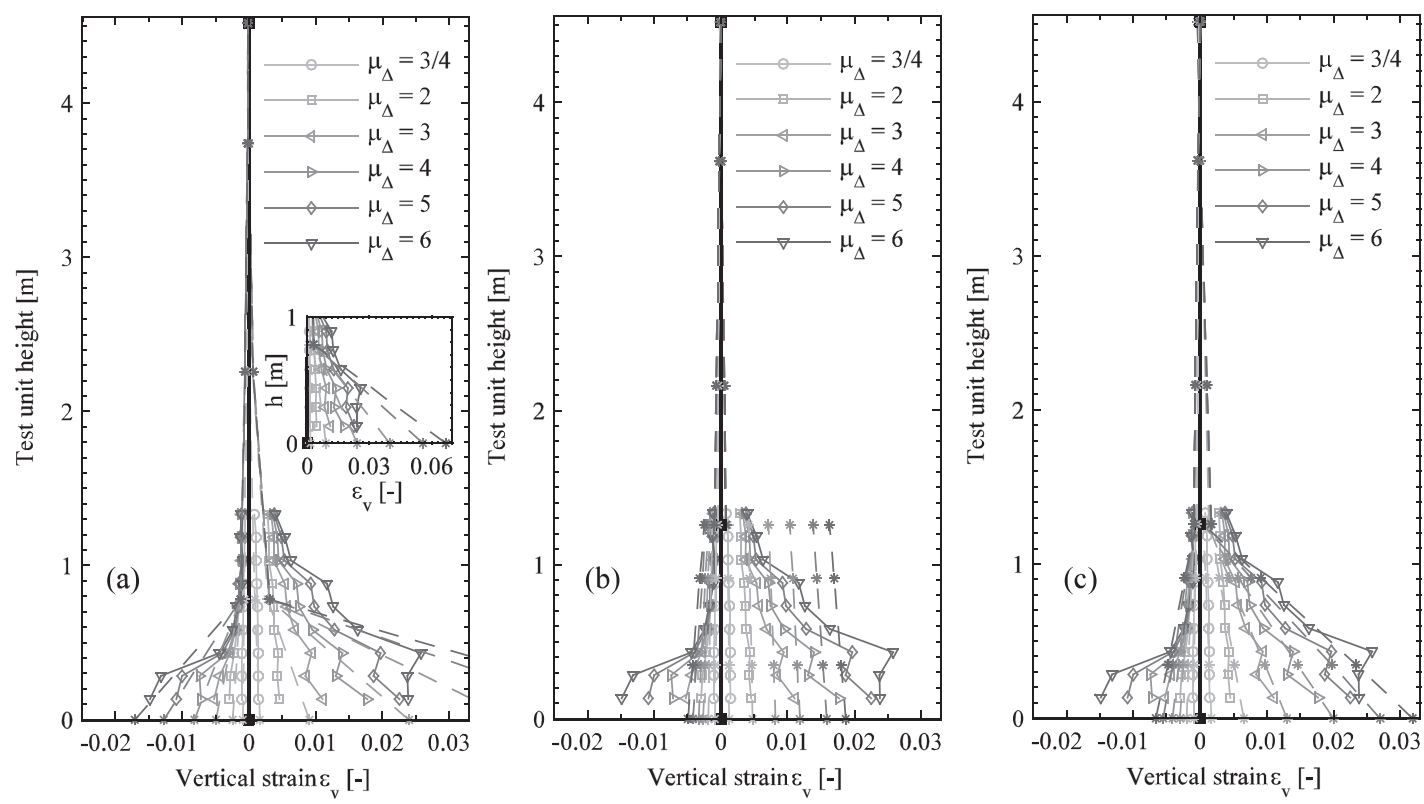

Figure 16. Experimental versus numerical vertical strain profiles of corner rebars for test WSH6 at positive ductility levels: (a) FB 5 IPs, (b) two-element DB/c and (c) two-element DB/ae models.

member. A smaller length for the base element would have improved the comparison at the price of worsening both the simulated curvatures and tensile strains. The good fit from the model FB 5IPs is however not a general rule, as confirmed by the results obtained in the previous subsection (Figure 11).

Finally, numerical and experimental base curvatures for the entire set of employed models and selected RC walls are compared in Table VI in terms of the average relative error $\left(\eta_{\phi b}^{a v g}\right)$, which is calculated at three distinct ductility levels. Similar to the results of the previous subsection (Table III), the DB/ae model with two elements per structural member generally leads to the highest precision. Once again, the worst results are obtained at the lowest ductility level $\left(\mu_{\Delta}=2\right)$, reaching a

Table VI. Numerical error in the calculation of the base curvature for all the models and selected test units tested by Dazio et al. [29] at different ductility levels.

\begin{tabular}{|c|c|c|c|c|c|c|c|}
\hline \multirow[b]{2}{*}{ Test } & \multirow{2}{*}{$\begin{array}{c}\text { Ductility } \\
\text { level } \\
\left(\mu_{\Delta}\right)\end{array}$} & \multicolumn{4}{|c|}{ Base curvature average relative error: $\boldsymbol{\eta}_{\phi \boldsymbol{b}}^{\boldsymbol{a v g}}=\frac{1}{2}$} & \multirow{2}{*}{$\frac{\left|1-\frac{\left(\phi_{b, a n}^{+}\right)}{\phi_{b, e x p}^{+}}\right|+\mid 1}{\text { DB/ae } 1 \text { ele. }}$} & \multirow{2}{*}{$\frac{\left.-\frac{\left(\phi_{b, a n}^{-}\right)}{\phi_{b, e x p}^{-}} \mid\right)}{\text {DB/ae } 2 \text { ele. }}$} \\
\hline & & FB 3IPs & FB 5IPs & $\mathrm{DB} / \mathrm{c} 1$ ele. & DB/c 2 ele. & & \\
\hline \multirow[t]{3}{*}{ WSH2 } & $\mu_{\Delta}= \pm 2$ & $17.1 \%$ & $16.8 \%$ & $55.1 \%$ & $7.7 \%$ & $40.0 \%$ & $0.2 \%$ \\
\hline & $\mu_{\Delta}= \pm 4$ & $34.1 \%$ & $58.3 \%$ & $68.3 \%$ & $27.4 \%$ & $58.1 \%$ & $10.1 \%$ \\
\hline & $\mu_{\Delta}= \pm 6$ & $56.4 \%$ & $15.7 \%$ & $80.0 \%$ & $52.2 \%$ & $73.2 \%$ & $22.7 \%$ \\
\hline \multirow[t]{3}{*}{ WSH3 } & $\mu_{\Delta}= \pm 2$ & $29.8 \%$ & $76.1 \%$ & $28.0 \%$ & $25.8 \%$ & $23.1 \%$ & $30.9 \%$ \\
\hline & $\mu_{\Delta}= \pm 4$ & $24.5 \%$ & $89.7 \%$ & $61.0 \%$ & $44.2 \%$ & $51.2 \%$ & $\mathbf{8 . 8} \%$ \\
\hline & $\mu_{\Delta}= \pm 6$ & $35.1 \%$ & $79.5 \%$ & $68.1 \%$ & $53.2 \%$ & $59.6 \%$ & $22.2 \%$ \\
\hline \multirow[t]{3}{*}{ WSH4 } & $\mu_{\Delta}= \pm 2$ & $20.4 \%$ & $37.2 \%$ & $52.8 \%$ & $27.4 \%$ & $41.7 \%$ & $3.0 \%$ \\
\hline & $\mu_{\Delta}= \pm 4$ & $35.2 \%$ & $70.2 \%$ & $67.0 \%$ & $44.4 \%$ & $58.5 \%$ & $15.0 \%$ \\
\hline & $\mu_{\Delta}= \pm 6$ & N/A & N/A & N/A & N/A & N/A & N/A \\
\hline \multirow[t]{3}{*}{ WSH5 } & $\mu_{\Delta}= \pm 2$ & $14.9 \%$ & $20.7 \%$ & $27.8 \%$ & $12.2 \%$ & $23.0 \%$ & $30.3 \%$ \\
\hline & $\mu_{\Delta}= \pm 4$ & $20.9 \%$ & $36.9 \%$ & $59.2 \%$ & $20.6 \%$ & $49.8 \%$ & $13.4 \%$ \\
\hline & $\mu_{\Delta}= \pm 6$ & $38.8 \%$ & $57.5 \%$ & $70.8 \%$ & $36.4 \%$ & $62.7 \%$ & $6.8 \%$ \\
\hline \multirow[t]{3}{*}{ WSH6 } & $\mu_{\Delta}= \pm 2$ & $12.1 \%$ & $63.0 \%$ & $34.4 \%$ & $9.2 \%$ & $21.7 \%$ & $17.9 \%$ \\
\hline & $\mu_{\Delta}= \pm 4$ & $25.0 \%$ & $86.2 \%$ & $60.9 \%$ & $40.9 \%$ & $53.0 \%$ & $6.1 \%$ \\
\hline & $\mu_{\Delta}= \pm 6$ & $19.4 \%$ & $124.8 \%$ & $59.1 \%$ & $36.6 \%$ & $50.9 \%$ & $10.7 \%$ \\
\hline
\end{tabular}

Note: minimum values of $\boldsymbol{\eta}_{\phi \boldsymbol{b}}^{a v g}$ for each test unit and ductility level are displayed in bold 
value of $\eta_{\phi b}^{a v g}$ around 30\%. This is sensibly smaller than the relative errors obtained with any of the other models, where the maximum $\eta_{\phi b}^{\text {avg }}$ ranged from $50 \%$ to $120 \%$.

\subsection{Limitations}

The validation examples presented in the previous sub-sections have shown that the DB/ae formulation can be effectively used in the simulation of the cyclic-nonlinear response of RC members with an encouraging match occurring also at the local strain level. Namely, it was shown that the linear curvature profile that develops in the plastic hinge region of a member in single bending due to tension shift effects can be captured by employing two DB/ae elements, and assigning a length for the bottom element equal to the extent of plasticity. This quantity is not usually available for engineering practice applications, and further research is yet required on the subject. A first approximation to estimate the extent of plasticity, which appears to be reasonably accurate for the present case studies, can be obtained by multiplying the 'equivalent plastic hinge length' as proposed by Priestley et al. [2] by a factor of two. This factor intends to account for the fact that the curvature profile is not constant but rather approximately linear within the inelastic region.

Additionally, as observed by Goodnight et al. [26], the length of plastification increases for increasing ductility demands, which is not accounted for in the present formulation. This contributes to a worse agreement between numerical and experimental local quantities for small ductility levels. Another limitation is that different lengths should be assigned to the bottom DB/ae element in order to optimally simulate tensile or compressive strain demand profiles. Because tension shift effects do not play a relevant role for compressive strains, a shorter length than the extent of plasticity would be required. However, this modelling issue is not specific to the present formulation as discussed by [26] in the context of plastic hinge analysis. Finally, it is noted that the entire set of test units used for validation showed a hardening behaviour of the structural member. Further research is required to investigate the softening response of the proposed $\mathrm{DB} /$ ae model.

\section{CONCLUSIONS}

Recent experimental tests on cantilever RC piers have confirmed that tension shift effects play an important role in the distribution of local level quantities such as strains and curvature profiles. Namely, linear curvatures are generated in the plastic hinge region due to inclined shear cracks, which intersect the elastic curvature profile at a certain height above the member foundation. These effects cannot be captured by current force-based formulations that satisfy equilibrium exactly, which consider only the effect of the moment gradient. Displacement-based formulations provide the natural framework to account additionally for tension shift effects as the linear plastic curvature distribution observed within the plastic region can be reproduced by imposing appropriate transversal displacement fields to the beam element.

However, the linear axial displacement profile used in classical displacement-based elements is a fundamental limitation to the accuracy of this approach when inelastic material behaviour is considered. The resulting axial forces are equilibrated only in an average sense, resulting in poor simulations of the experimental force-displacement response, as well as curvature and strain profiles.

In view of the above, this paper presents a displacement-based element that strictly satisfies axial equilibrium. An intra-element iterative scheme that automatically adjusts the axial strain profile is implemented to attain constant axial forces in all integration points, and equal to the applied axial load. The curvature profiles are instead kept linear as in classical displacement-based elements, although they result quantitatively different on account of the axial equilibrating procedure. The principle of virtual work is employed to obtain the element basic forces and a consistent stiffness matrix.

The axially equilibrated displacement-based element is validated against two sets of cyclic tests on RC cantilever piers and walls. Assuming an appropriate member discretization, it provides accurate results in terms of global and local scale response. Namely, the simulation of experimental curvatures and strains shows a significant improvement when compared with models using classical force-based or displacement-based elements. As an example, when base curvatures over different 


\section{AXIALLY EQUILIBRATED DISPLACEMENT-BASED ELEMENT FOR RC MEMBERS}

ductility levels are considered, the model using the proposed formulation provides the best estimation in about $80 \%$ of the cases. Nevertheless, due to the different length of the plastic region over which tensile and compressive strains develop, different levels of accuracy are obtained for these quantities. The improved predictions come at the cost of slightly increased computational time with respect to the classical displacement-based formulation.

\section{ACKNOWLEDGEMENTS}

The research presented in this paper is part of a project funded by the Swiss Federal Roads Office (FEDRO) under project number AGB 2015/002. Additionally, the authors would like to thank Dr. Michele Godio and Dr. Nuno Pereira as well as two anonymous reviewers for their insightful suggestions and inspiring comments.

\section{REFERENCES}

1. Berry MP, Lehman DE, Lowes LN. Lumped-plasticity models for performance simulation of bridge columns. ACI Structural Journal 2008; 105:270-279.

2. Priestley MJN, Calvi GM, Kowalsky MJ. Displacement-based Seismic Design of Structures. IUSS Press, 2007.

3. Zhao J, Sritharan S. Modeling of strain penetration effects in fiber-based analysis of reinforced concrete structures. ACI Structural Journal 2007; 104:133-141.

4. Monti G, Spacone E. Reinforced concrete fiber beam element with bond-slip. Journal of Structural Engineering 2000; 654-661.

5. Spacone E, Filippou FC, Taucer FF. Fibre beam-column model for non-linear analysis of R/C frames: Part 1. Formulation. Earthquake Engineering and Structural Dynamics 1996; 25:711-725.

6. Yazgan U, Dazio A. Simulating maximum and residual displacements of RC structures: I. Accuracy, Earthquake Spectra 2011; 27:1187-1202.

7. Yazgan U, Dazio A. Simulating maximum and residual displacements of RC structures: II. Sensitivity, Earthquake Spectra 2011; 27:1203-1218.

8. Almeida JP, Das S, Pinho R. Adaptive force-based frame element for regularized softening response. Computers and Structures 2012; 102-103:1-13.

9. Scott MH, Hamutcuoglu OM. Numerically consistent regularization of force-based frame elements. International Journal for Numerical Methods in Engineering 2008; 76:1612-1631.

10. Almeida JP, Tarquini D, Beyer K. Modelling approaches for inelastic behaviour of RC walls: multi-level assessment and dependability of results. Archives of Computational Methods in Engineering 2016; 23:69-100.

11. SeismoSoft, SeismoStruct—a computer program for static and dynamic nonlinear analysis of framed structures, (2013).

12. McKenna F, Fenves GL, Scott MH, Jeremic B. Open System for Earthquake Engineering Simulation (OpenSees). California, USA: Berkeley, 2000.

13. Calabrese A, Almeida JP, Pinho R. Numerical issues in distributed inelasticity modeling of RC frame elements for seismic analysis. Journal of Earthquake Engineering 2010; 14:38-68.

14. Goodnight JC, Kowalsky MJ, Nau JM. Effect of load history on performance limit states of circular bridge columns. Journal of Bridge Engineering 2013; 18:1383-1396.

15. Hines EM. Seismic performance of hollow rectangular reinforced concrete bridge piers with confined corner elements. PhD Thesis, Univ. Calif. (2002).

16. Hose YD, Seible F, Priestley MJN. Strategic relocation of plastic hinges in bridge columns. Struct. Syst. Res. Proj. 97/05, Univ. Calif. 1997.

17. Chai YH, Priestley MJN, Seible F. Flexural retrofit of circular reinforced concrete bridge columns by steel jacketing: experimental studies. Struct. Syst. Res. Proj. 91/06, Univ. Calif. 1991; 151.

18. J.C. Goodnight, M.J. Kowalsky, J.M. Nau, A new look at strain limits and plastic hinge lengths for reinforced concrete bridge columns, in: 10th U.S. Natl. Conf. Earthq. Eng., Anchorage, 2014.

19. Izzuddin B, Karayannis C, Elnashai A. Advanced nonlinear formulation for reinforced concrete beam-columns. Journal of Structural Engineering 1994; 120:2913-2934.

20. De Souza R. Force-based finite element for large displacement inelastic analysis of frames. Ph.D. Diss. Dep. Civ. Environ. Eng. Univ. California, Berkeley, USA. 2000.

21. Almeida JP, Tarquini D. SAGRES: Software for Analysis of GRadient Effects in Structures. Progr. Dev. Matlab. 2016.

22. Popovics S. A numerical approach to the complete stress-strain curve of concrete. Cement and Concrete Research 1973; 3:583-599.

23. Goodnight JC, Feng Y, Kowalsky MJ, Nau JM. The effects of load history and design variables on performance limit states of circular bridge columns. Volume 2: Experimental Observations, Report, Alaska Dep. Transp. Public Facil. Res. 40002015.

24. Scott MH, Fenves GL. Plastic hinge integration methods for force-based beam-column elements. Journal of Structural Engineering 2006; 132:244-252. 


\section{TARQUINI, J. P. ALMEIDA AND K. BEYER}

25. Neuenhofer A, Filippou FC. Evaluation of nonlinear frame finite-element models. Journal of Structural Engineering 1997; 123:958-966.

26. Goodnight JC, Kowalsky MJ, Nau JM. Modified plastic-hinge method for circular RC bridge columns. Journal of Structural Engineering 2016. DOI:10.1061/(ASCE)ST.1943-541X.0001570.

27. Menegotto M, Pinto PE. Method of analysis for cyclically loaded RC plane frames including changes in geometry and non-elastic behaviour of elements under combined normal force and bending. in: IABSE Symp. Resist. Ultim. Deform. Struct. Acted by Well Defin. Repeated Loads-Final Rep, 1973.

28. Mander JB, Priestley MJN, Park R. Theoretical stress-strain model for confined concrete. Journal of Structural Engineering 1988; 114:1804-1826.

29. Dazio A, Beyer K, Bachmann H. Quasi-static cyclic tests and plastic hinge analysis of RC structural walls. Engineering Structures 2009; 31:1556-1571.

30. Park R. Ductility evaluation from laboratory and analytical testing. in: Proc. 9th World Conf. Earthq. Eng, 1988.

31. Hines EM, Restrepo JI, Seible F. Force-displacement characterization of well-confined bridge piers. ACI Structural Journal 2004; 101:537-548.

32. Beyer K, Dazio A, Nigel Priestley MJ. Shear deformations of slender reinforced concrete walls under seismic loading. ACI Structural Journal 2011; 108:167-177.

33. Mergos PE, Beyer K. Modelling shear-flexure interaction in equivalent frame models of slender reinforced concrete walls. The Structural Design of Tall Special Buildings 2014; 23:1171-1189. 